\title{
Validating a new methodology for optical probe design and image registration in fNIRS studies
}

\author{
Sobanawartiny Wijeakumar ${ }^{\text {a, }}$, John P. Spencer ${ }^{a}$, Kevin Bohache ${ }^{a}$, David A. Boas ${ }^{c}$, Vincent A. Magnotta ${ }^{b}$ \\ a University of Iowa, Department of Psychology and Delta Center, Iowa City 52242, IA, USA \\ ${ }^{\mathrm{b}}$ University of Iowa, Department of Radiology and Delta Center, Iowa City 52242, IA, USA \\ c Athinoula A. Martinos Center for Biomedical Imaging, Department of Radiology, Massachusetts General Hospital, Harvard Medical School, Charlestown, MA, USA
}

\section{A R T I C L E I N F O}

\section{Article history:}

Accepted 11 November 2014

Available online 22 November 2014

\section{Keywords:}

Functional near infrared spectroscopy

Probe geometry

Optode co-registration

Visual working memory

AtlasViewerGUI

\begin{abstract}
A B S T R A C T
Functional near-infrared spectroscopy (fNIRS) is an imaging technique that relies on the principle of shining near-infrared light through tissue to detect changes in hemodynamic activation. An important methodological issue encountered is the creation of optimized probe geometry for fNIRS recordings. Here, across three experiments, we describe and validate a processing pipeline designed to create an optimized, yet scalable probe geometry based on selected regions of interest (ROIs) from the functional magnetic resonance imaging (fMRI) literature. In experiment 1 , we created a probe geometry optimized to record changes in activation from target ROIs important for visual working memory. Positions of the sources and detectors of the probe geometry on an adult head were digitized using a motion sensor and projected onto a generic adult atlas and a segmented head obtained from the subject's MRI scan. In experiment 2, the same probe geometry was scaled down to fit a child's head and later digitized and projected onto the generic adult atlas and a segmented volume obtained from the child's MRI scan. Using visualization tools and by quantifying the amount of intersection between target ROIs and channels, we show that out of 21 ROIs, 17 and 19 ROIs intersected with fNIRS channels from the adult and child probe geometries, respectively. Further, both the adult atlas and adult subject-specific MRI approaches yielded similar results and can be used interchangeably. However, results suggest that segmented heads obtained from MRI scans be used for registering children's data. Finally, in experiment 3, we further validated our processing pipeline by creating a different probe geometry designed to record from target ROIs involved in language and motor processing.
\end{abstract}

(c) 2014 Elsevier Inc. All rights reserved.

\section{Introduction}

Functional near-infrared spectroscopy (fNIRS) is an imaging technique that relies on the principle of shining light in the near-infrared range (695-1000 $\mathrm{nm}$ ) through tissue. fNIRS systems measure the absorption and scattering of photons as light passes through, providing a quantitative measurement of blood oxygenation. In particular, light at the lower end and higher end of the near-infrared spectrum is selectively absorbed by oxy and de-oxy hemoglobin, respectively. As chromophore concentration levels change as a function of localized oxygen extraction, the extent of activation can be estimated from a difference in the amount of light entering tissue and light that is collected by a detector placed at the surface, some distance away.

fNIRS is portable, cheap, has better temporal resolution, and is much less susceptible to motion artifacts as compared to functional magnetic resonance imaging. However, fNIRS offers a lower spatial resolution and can only record from within a few centimeters of the cortex. Despite

\footnotetext{
* Corresponding author at: E1 Seashore Hall, Department of Psychology, University of Iowa, Iowa City, IA 52245, USA.

E-mail address: sobanawartiny-wijeakumar@uiowa.edu (S. Wijeakumar).
}

these limitations, fNIRS is a more feasible neuroimaging tool when examining cortical function within certain cohorts. For instance, fNIRS has been used to study changes in cortical activation across development and with atypical populations. The thinner scalp and skull in neonates and children allow for recording from deeper cortical structures (Gervain et al., 2011; Lin et al., 2013). Given these advantages, fNIRS has been extensively used to investigate the neural mechanisms underlying a host of topics including visual processing (Wijeakumar et al., 2012a,b), auditory processing (Bortfeld et al., 2007; Fava et al., 2013), processing of language and speech (Sato et al., 2012; Yoo and Lee, 2013), motor function (Gagnon et al., 2012; Kuboyama et al., 2004), and other cognitive domains including working memory (Buss et al., 2014; Jausovec and Jausovec, 2012; Sato et al., 2013).

Although several cross-validation studies have demonstrated that fNIRS is an effective neuroimaging tool (Cui et al., 2011; Huppert et al., 2006), there are no standard procedures for designing probe geometries-that is, the distribution of sources and detectors on the scalp-to optimize fNIRS data collection. Probe geometry is a critical factor in fNIRS studies because every element from the start of data collection through analysis is contingent upon the placement of sources and detectors with respect to the scalp surface and the underlying 
anatomy. If placement is not consistent from subject-to-subject and session-to-session, variation in functional data may not be reflective of differences in underlying neural systems; instead, it could simply be a product of improper placement of the probe itself and resultant recordings from different parts of cortex. Moreover, if the probe geometry is not optimized, it is possible to miss target regions of interest (ROIs) within cortex.

One approach to probe placement commonly used in fMRI-NIRS validation studies is to place MRI visible capsules (e.g. Vitamin E) adjacent to the sources and detectors to identify the areas of the cortex recorded from (Huppert et al., 2006; Kovelman et al., 2009). A few studies have also used digitization techniques to co-register the exact positions of the sources and detectors to an MRI scan (Whalen et al., 2008). This is not a generic solution for fNIRS studies, however, because it is prohibitively expensive to use MRI in conjunction with fNIRS for every study and every cohort.

In the present report, we describe a processing pipeline using freely available software to create an optimized ${ }^{1}$, yet scalable probe geometry. This methodological paper highlights a set of tools (a) to systematically select a group of target ROIs from the neuroimaging literature relevant to a study, (b) to create a preliminary probe geometry based on the selected ROIs, (c) to create a protocol for digitizing scalp landmarks and positions of sources and detectors, (d) to transform and visualize the preliminary positions of sources, detectors, and ROIs on an adult atlas or a segmented head generated from an subject-specific MRI scan, (e) to estimate and create sensitivity distributions of photon migration (Monte Carlo simulations) through scalp and cortex for each channel, and (f) to visualize and quantify the amount of overlap between these sensitivity distributions and the target ROIs. We describe how users can iterate through these steps to refine initial probe geometry, and we demonstrate the validity of the approach across three experiments. In Experiment 1, we developed an adult probe geometry based on ROIs for a visual working memory (VWM) study. In Experiment 2, the same probe geometry was scaled down to fit a child's head. Finally, in Experiment 3, we extended the protocol to a completely different probe geometry designed to investigate motor and language processing in the adult brain. We conclude by discussing how this pipeline might be refined in future work and how these tools might be used in analysis of fNIRS data, moving from channel-wise analyses to image-based analyses.

\section{Experiment 1}

The aim of this experiment was to develop and validate a new pipeline to optimize a probe geometry design for studies examining VWM. VWM is a good test case for evaluating the new pipeline for several reasons. First, it is a cognitive process of interest with dramatic individual differences and links to measures of general cognitive function including fluid intelligence and pathopsychology (Alloway, 2007; Steele et al., 2007). Second, the ROIs identified in fMRI studies of VWM are positioned relatively close to the cortical surface and, thus, are detectable using fNIRS. Finally, a recent study demonstrated that a fronto-parietal VWM network common in adult fMRI studies is also actively engaged by 3- and 4-year-olds in a VWM task, raising the possibility of using fNIRS to study the early development of this critical cognitive system (Buss et al., 2014). To understand the development of VWM, however, it is necessary to design a probe geometry that can be

\footnotetext{
${ }^{1}$ Note that the pipeline we describe does not yield an optimal probe geometry in a mathematical sense; rather, we use the term 'optimal' colloquially throughout. Given the vast degrees of freedom in the initial geometry on the scalp and the need to express the ultimate geometry in a way that can be reproduced across head sizes, we did not pursue an optimal mathematical solution. Future efforts in this direction could certainly build on the pipeline reported here.
}

scaled over development to record from the target ROIs. We describe the processing pipeline below, along with key results from each step.

\section{Methods and results}

The steps involved in designing optimal probe geometry are outlined below. Fig. 1 shows a flowchart of the pipeline. It should be noted that pipeline was iterative and terminated when an optimized probe geometry was obtained. The observations reported in the following sections are the results from the final optimized probe geometry design, although we also discuss how an initial geometry was modified as we iterated through the pipeline.

\section{Regions of interest (Step 1)}

We used the Pubmed database to search for literature relevant to our study of visual working memory. The keywords for the search included 'visual working memory, feature working memory, change detection, visual short term memory, visual short term recall, and functional magnetic resonance imaging'. We only included studies that investigated normal populations. The literature search revealed 8 fMRI studies on visual working memory that met these criteria (see Table 1 ).

Most of the manuscripts reported the coordinates of the ROIs in MNI space. Those that were reported in Talaraich space were converted to MNI space using the Lancaster transform (Laird et al., 2010) available in GingerALE software (GingerALE Version 2.3, BrainMap, Research Imaging Institute of University of Texas Health Center, San Antonio, Texas, U.S.A.). A total of 92 ROIs were identified. Next, pairs of ROIs that were a distance of $1 \mathrm{~cm}$ or less from each other were averaged and presented as a single ROI. This process was implemented in two stages: (1) We calculated the Euclidian distances between ROI pairs for ROIs with the same name that were reported across different contrasts, but within the same paper. Sets of ROIs that were separated by a distance of less than $1 \mathrm{~cm}$ were averaged together. These 'unique' ROIs were added to the full set across studies. (2) The same process was repeated with the full set of ROIs across studies. The final list consisted of 21 ROIs, which are shown, in Table 2. Note that it is not necessary to average ROIs positioned within $1 \mathrm{~cm}$ of each other. We did this here, in part, to simplify presentation. It is also the case, however, that the spatial resolution of fNIRS is such that the difference in the amount of intersection of a NIRS channel and two ROIs positioned within $1 \mathrm{~cm}$ of each other is likely to be negligible.

The next step was to take the ROIs and create a target brain image. For each ROI, we created a sphere of radius $6 \mathrm{~mm}$ at the $\mathrm{x}, \mathrm{y}$, and $\mathrm{z}$ MNI coordinates upon an averaged T1 $(n=17)$ base image using the Analysis of Functional Neurolmages (AFNI, NIMH Bethesda, MD, U.S.A.) software (voxel size of $2 \times 2 \times 2 \mathrm{~mm}$ ).

In the current experiment, we chose a male adult ( 26 years old) with a head circumference of $58 \mathrm{~cm}$. At this circumference, we used a sourcedetector distance of $29 \mathrm{~mm}$. This source-detector distance was selected based on a mapping of head circumference to source-detector distances over development, which we describe in Experiment 2 (see Table 4). We used caps manufactured by EasyCAP (Brain Products $\mathrm{GmBH}$, Germany), originally designed for recording electroencephalograms. These caps can be fitted with customized grommets (available from TechEn, Inc.) to secure the fNIRS optodes to the scalp.

\section{Preliminary probe geometry (Step 2)}

The final set of ROIs was transformed to a generic adult atlas and visualized using 3D Slicer, a free and open source software package (Fedorov et al., 2012) (http://www.slicer.org). Based on the distribution of ROIs, preliminary probe geometry was created to initiate the pipeline. We used the ROI image in 3D Slicer as a guide and designed a geometry that we thought would provide good coverage of the ROIs. In particular, we observed that our ROIs were distributed across the left and right frontal and parieto-occipital cortices. Given this wide distribution, we 


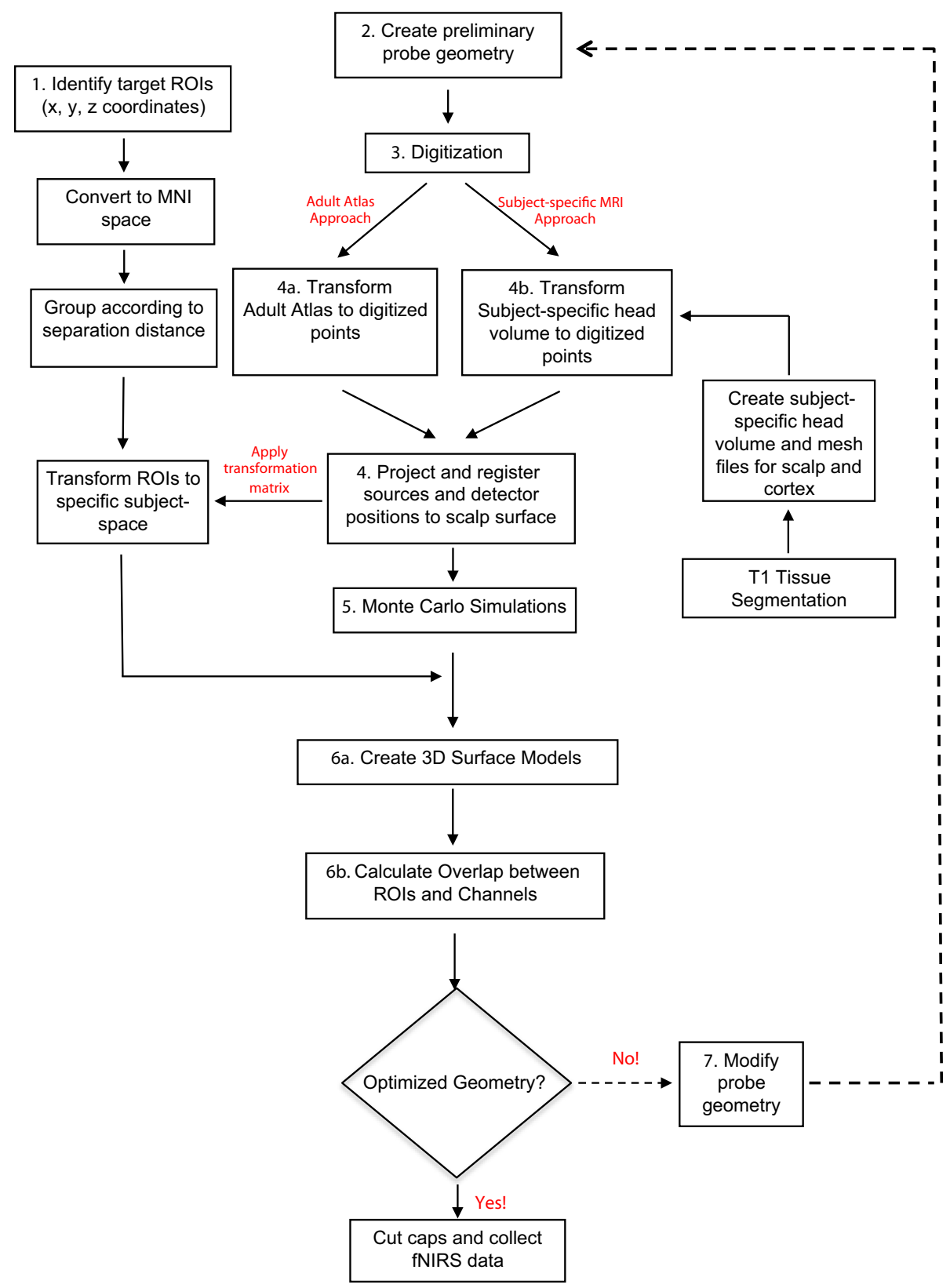

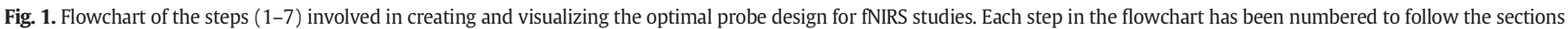
within the text.

decided to divide our probe geometry such that we obtained four 'quadrants' covering the left and right frontal and parieto-occipital cortices respectively. Next, we decided to opt for an equal number of sources and detectors in each quadrant resulting in 3 sources and 5 detectors per quadrant (we had a total of 12 sources and 20 detectors available for this study).

Table 1

Journal articles from which ROIs for visual working memory were obtained (for Experiments 1 and 2).

\begin{tabular}{|c|c|c|c|}
\hline No. & Title & Authors & Year \\
\hline 1 & The role of the parietal cortex in visual feature binding & Shafritz KM, Gore JC \& Marois R. & 2002 \\
\hline 2 & Neural correlates of visual working memory: fMRI amplitude predicts task performance & Pessoa L, Gutierrez E, Bandettini PA \& Ungerleider LG & 2002 \\
\hline 3 & Capacity limit of visual short-term memory in human posterior parietal cortex & Todd JJ \& Marois R & 2004 \\
\hline 4 & $\begin{array}{l}\text { "What" and "where" in the Intraparietal Sulcus: an fMRI study of object identity } \\
\text { ad location in visual short-term memory }\end{array}$ & Harrison A, Jolicoeur P \& Marois R & 2010 \\
\hline 5 & Neural correlates of change detection and change blindness in working memory task & Pessoa L \& Ungerleider LG & 2004 \\
\hline 6 & $\begin{array}{l}\text { visual short-term memory load suppresses temporo-parietal junction activity and } \\
\text { induces inattentional blindness }\end{array}$ & Todd JJ, Fougnie D \& Marois R & 2005 \\
\hline 7 & Dissociable neural mechanisms supporting visual short-term memory for objects & Xu Y \& Chun MM & 2006 \\
\hline 8 & Mechanism for top-down control of working memory capacity & Edin F, Klingberg T, Johansson P, McNab F, Tegner J \& Compte A & 2008 \\
\hline
\end{tabular}


Table 2

List of ROIs used in the design of the probe for visual working memory (Experiments 1 and 2). Note that the serial numbering for each ROI is used for identification in later tables.

\begin{tabular}{lllrrr}
\hline \multirow{2}{*}{ No. } & Regions of interest & Hemisphere & \multicolumn{3}{c}{ MNI coordinates } \\
\cline { 4 - 6 } & & & $\mathrm{x}$ & $\mathrm{y}$ & $\mathrm{z}$ \\
\hline \multirow{2}{*}{1} & Superior Intraparietal Sulcus (sIPS) & Right & 25 & -61 & 51 \\
2 & Intraparietal Sulcus (IPS) & Right & 30 & -73 & 38 \\
3 & Intraparietal Sulcus (IPS) & Left & -21 & -65 & 46 \\
4 & Anterior Intraparietal Sulcus (aIPS) & Right & 40 & -36 & 38 \\
5 & Anterior Intraparietal Sulcus (aIPS) & Left & -40 & -44 & 42 \\
6 & Ventral Occipital Cortex (VOC) & Right & 42 & -75 & -3 \\
7 & Ventral Occipital Cortex (VOC) & Left & -42 & -71 & 6 \\
8 & Dorsolateral Prefrontal Cortex (DLPFC) & Right & 45 & 42 & 21 \\
9 & Dorsolateral Prefrontal Cortex (DLPFC) & Left & -42 & 41 & 20 \\
10 & Superior Frontal Gyrus (SFG) & Left & -1 & 18 & 51 \\
11 & Inferior Frontal Gyrus (IFG) & Right & 35 & 22 & 4 \\
12 & Inferior Frontal Gyrus (IFG) & Left & -31 & 23 & -1 \\
13 & Frontal Eye Fields (FEF) & Right & 29 & -2 & 52 \\
14 & Frontal Eye Fields (FEF) & Left & -25 & -3 & 54 \\
15 & Middle Frontal Gyrus (MFG) & Right & 49 & 6 & 31 \\
16 & Middle Frontal Gyrus (MFG) & Left & -43 & 3 & 29 \\
17 & Anterior Cingulate Cortex (ACC) & Left & -5 & 30 & 27 \\
18 & Occipital (OCC) & Right & 34 & -87 & 13 \\
19 & Occipital (OCC) & Left & -34 & -85 & 14 \\
20 & Temporo-Parietal Junction (TPJ) & Right & 62 & -46 & 26 \\
21 & Temporo-Parietal Junction (TPJ) & Left & -58 & -49 & 28 \\
\hline & & & &
\end{tabular}

A step-by-step illustration of the process of drawing the left frontal probe geometry is shown in Fig. 2. Sources and detectors are shown in red and blue respectively. Each source and each detector position were aligned to the 10-20 System of Electrode Placement, either directly above a 10-20 landmark or geometrically positioned relative to two or more landmarks. This is important because it ensures that the geometry can be reliably reproduced if multiple caps are used in the study. This also facilitates scaling the geometry to different head sizes.

First, sources S1 and S3 were placed at 10-20 positions F7 and F3, respectively, because the $\mathrm{ROI}$ image in $3 \mathrm{D}$ Slicer indicated that many of the left frontal ROIs were near these landmarks. A circle was drawn around each one (radius $=29 \mathrm{~mm}$ ) and detectors D1 and D2 were placed at the intersections of these two circles (Fig. 2A). This created four channels (S1-D1, S1-D2, S3-D1, S3-D2) with the desired sourcedetector separation $(29 \mathrm{~mm})$ via the judicious placement of two sources and two detectors. Next, circles (radius $=29 \mathrm{~mm}$ ) were drawn around $\mathrm{AFz}$ and Fz. Detector D5 was placed at the intersection of the circle around S3 and the circle around AFz. Detector D4 was placed at the intersection of the circle around S3 and the circle around Fz (Fig. 2B). These positions created channels over more medial regions of the left frontal cortex near ROIs in the 3D Slicer image.

The remaining left frontal ROIs were in more posterior regions of the left frontal cortex. Thus, we positioned our remaining source posterior to $S 3$ at a position where we could re-purpose D1 and D4. Specifically, circles were drawn around detectors D1 and D4, and source S2 was placed at the posterior intersection of these circles (Fig. 2C). This created two new channels - S2-D1 and S2-D4 - from the placement of a single additional source. Finally, inspection of the ROIs in the 3D Slicer image suggested that we should place our remaining detector in a more medial and posterior position to capture, for instance, activation near the frontal eye fields. To do this, a circle was drawn around source S2 and a line was drawn connecting source S2 and landmark Cz. Detector D3 was placed within range of source S2 along this line (Fig. 2D). Fig. 2E shows one of the final stages in the drawing of the left frontal probe geometry on the actual cap. Fig. $2 \mathrm{~F}$ shows a picture from one of the final stages in the drawing of the left parietal probe geometry. Note that this section outlines the process leading to an optimal geometry. The final left frontal geometry (after modifications) is discussed in Step 7 (Modifying probe geometry as needed section) and shown in Fig. 6.
Digitization (Step 3)

Once we obtained a probe geometry that occupied all our sources and detectors, we proceeded to digitize these points as an adult subject wore the cap such that the 10-20 landmark positions marked on the cap were aligned precisely on the subject's head. Next, a Polhemus Patriot ${ }^{\mathrm{TM}}$ Motion Tracking system (Colchester, VT, U.S.A.) was used to digitize the scalp landmarks (nasion, inion, $\mathrm{Cz}$, and left and right peri-auricular points) and the optode positions. The reference was placed at $\mathrm{Cz}$ and a stylus was used to digitize each point. It is crucial that a cap that matches the subject's head circumference be chosen. Further, it is important that the cap is positioned correctly on the head. To verify this, we measured the distances from $\mathrm{Cz}$ on the subject's scalp to the left and right periauricular points, nasion, and inion, and then re-checked these measurements using point $\mathrm{Cz}$ on the cap after cap placement.

A template of the probe geometry was created in 2D space using SDGui software in HOMER2 (Huppert et al., 2009). Twelve sources and twenty detectors were used in the probe geometry ( 3 sources and 5 detectors per quadrant) resulting in 36 channels ( 9 per quadrant; see Fig. 2).

Transforming adult atlas and subject-specific segmented head (from MRI scan) to digitized points (Step 4)

The next step in the pipeline was to transform some representation of the head and brain to the digitized points from Step 3. The digitized points could be used to transform a generic adult atlas readily available within AtlasViewerGUI (available within HOMER2: www.nmr.mgh. harvard.edu/PMI/resources/homer2). It is also possible to transform a subject-specific head volume obtained by segmenting tissue types from an MRI scan. These two options are discussed in the following sections.

Adult atlas approach (Step 4a). For the adult atlas approach, a generic adult atlas (available in the AtlasViewerGUI program in HOMER2) was constructed from a high-resolution digital phantom 'Colin27' (Collins et al. 1988). This is a standard atlas used in the MRI community and the segmented volume of the head structure as well as surfaces of the brain and head are readily available on-line (Custo et al., 2010).

Subject-specific MRI approach (Step 4b). To use a subject-specific head volume, structural information was obtained from a T1 scan of an adult brain collected on a Siemens 3T TIM Trio scanner (3D MPRAGE: $\mathrm{TI}=1200 \mathrm{~ms}, \mathrm{TE}=3 \mathrm{~ms}, \mathrm{TR}=2400 \mathrm{~ms}$, flip angle $=8^{\circ}$, matrix $=$ $256 \times 224 \times 160, \mathrm{FOV}=256 \times 224 \times 160, \mathrm{BW}=220 \mathrm{~Hz} /$ pixel, $\mathrm{iPAT}=2$ ).

Freesurfer was used to segment the T1-weighted scan from the adult into separate volumes of gray matter, white matter, and cerebro-spinal fluid. Voxels representing brain tissue (gray and white matter) and scalp voxels were identified and assigned unique values. These volumes (i.e., tissue and scalp) were then converted to 3D mesh surfaces and merged together to create the subject-specific 3D head volume in the same coordinate system as that of the generic adult atlas.

\section{AtlasViewer and Monte Carlo simulations (Step 5)}

Once the head model had been transformed to the digitized points, the points were projected to the scalp by using a relaxation algorithm described by Cooper and colleagues (Cooper et al., 2012). The images were visually verified to ensure that the points were projected correctly onto the scalp (errors in the measurement of the scalp landmarks could produce an invalid projection). Further, the positions of the sources and detectors were checked to make sure they were symmetric across the left and right hemispheres (asymmetries could indicate mis-alignment of the cap on the subject's head). Fig. 3 shows the digitized points from the adult probe geometry registered onto an adult atlas. Red and blue circles represent sources and detectors and their connections are represented in yellow. 
A

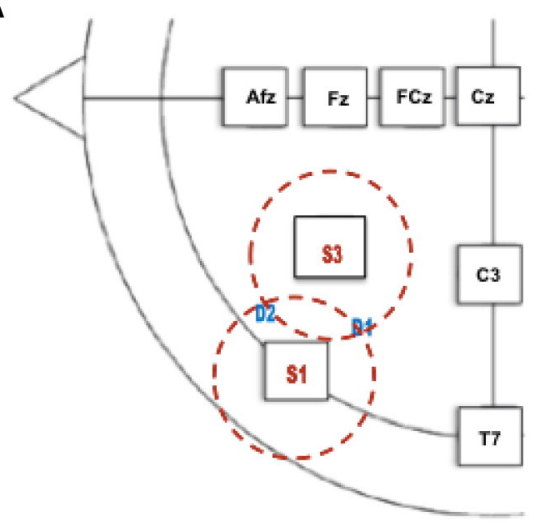

C

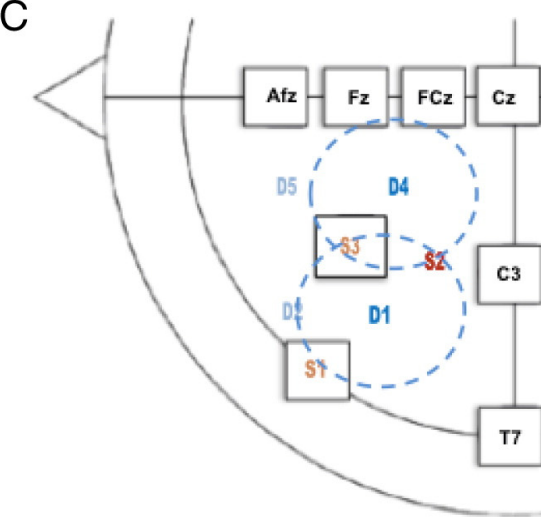

E

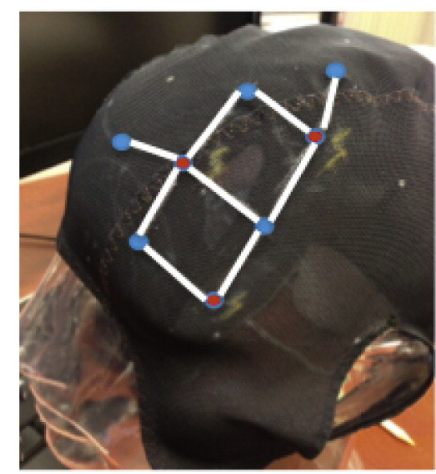

B

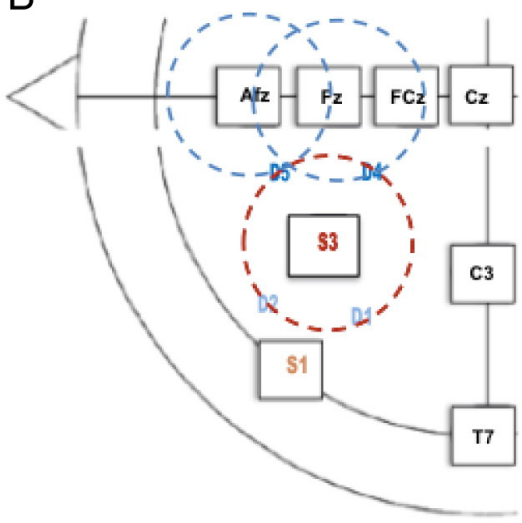

D

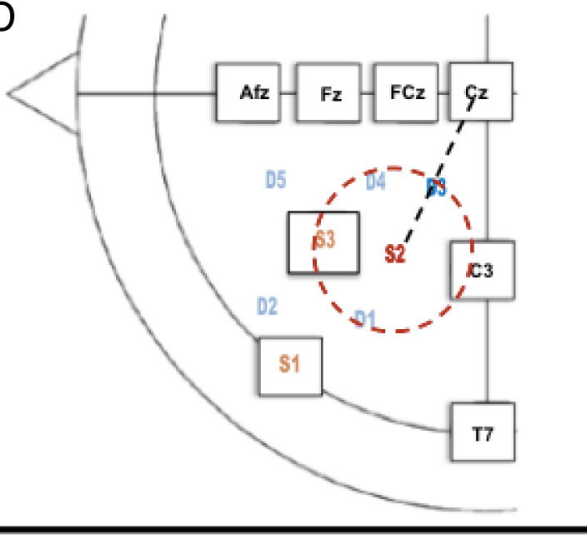

$\mathrm{F}$

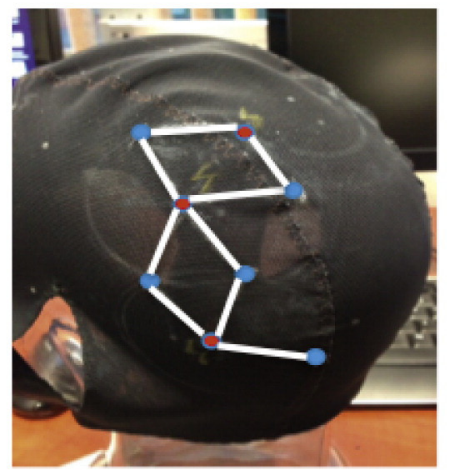

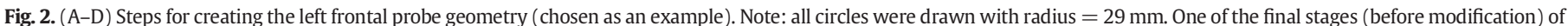

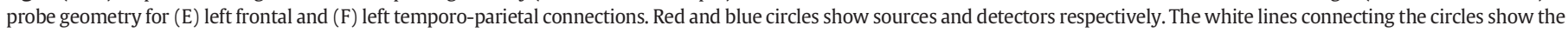
source-detector connections.

AtlasviewerGUI (HOMER2, Massachusetts General Hospital/Harvard Medical School, MA, U.S.A.) was used to run Monte Carlo simulations based upon a GPU-dependent Monte Carlo algorithm (Fang and Boas, 2009; Selb et al., 2014) to create measurement sensitivity distributions for each channel of the probe geometry. The absorption and reduced scattering coefficients for white matter and gray matter were $0.0178 \mathrm{~mm}^{-1}$ and $1.25 \mathrm{~mm}^{-1}$ respectively. The absorption and reduced scattering coefficients for extra-cerebral tissues were $0.0159 \mathrm{~mm}^{-1}$ and $0.8 \mathrm{~mm}^{-1}$ respectively.

The output from the Monte Carlo simulations yields a sensitivity distribution for each of the 36 channels reflecting the sensitivity of that channel to detecting changes in the cortical absorption of nearinfrared light. Fig. 4 shows the sensitivity distributions for the adult subject using the adult atlas approach (Fig. 4 top panels) and the adult subject-specific MRI approach (Fig. 4 bottom panels). As evident in both figures, the probe geometry manages to capture large parts of the fronto-parietal areas that are activated during VWM tasks.

The surface visualization in Fig. 4 shows the broad regions of the cortex that will be recorded from using this specific probe geometry, but it is not possible to determine whether the sensitivity distributions intersect with the target ROIs. Thus, the next step in the processing pipeline is to quantify the amount of intersection, if any, between the sensitivity distributions and the target ROIs by moving to a volumetric representation.

Evaluation of probe geometry (Step 6)

The next step in the processing pipeline was to evaluate the probe geometry in the context of the photon migration results: does the 


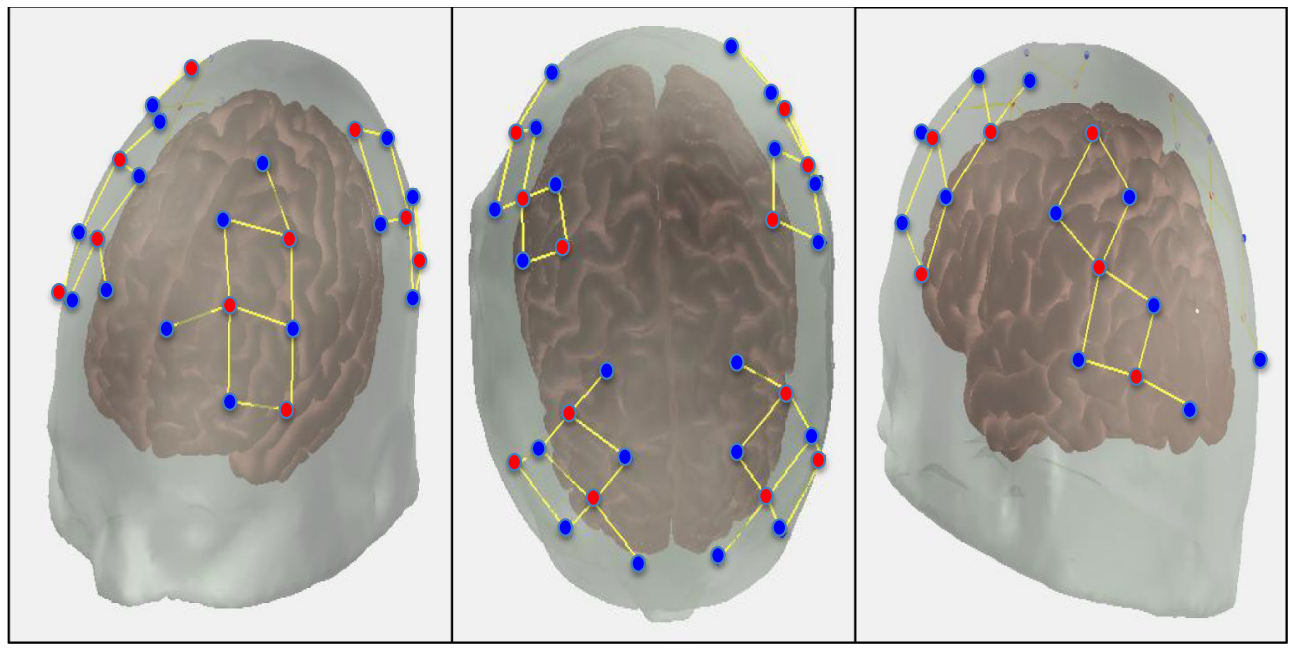

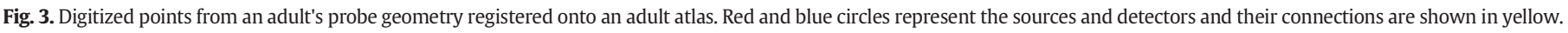

given geometry produce an overlap between the target ROIs and the sensitivity distributions? We do this in two steps below. First, we visualize the ROIs and sensitivity distributions. Second, we quantify the overlap between each ROI and each channel.

Visualization in Slicer (Step 6a). To assess the effectiveness of the probe geometry, we want to visualize the sensitivity distributions in the same image space as the target ROIs. An affine transformation was used to define the correspondence between the subject-specific head volume and the averaged T1 upon which the ROIs were created, using the BRAINSFit registration tool (available in Slicer). The transformation matrix obtained from this step was then used to resample and transform the generated ROI spheres to the subject-specific brain volume.

Surface representations of the head (scalp), sensitivity distributions of channels, and ROIs were created using the ModelMaker module in Slicer. The resulting surfaces were decimated and smoothed for visualization. Note that results discussed in the next two sections are based upon the final probe geometry. Fig. 5 shows left and right views of 3D surface models of the skull, sensitivity distributions (in gray and yellow), and several ROIs (blue) of the right hemisphere for the adult atlas approach (top panels) and adult subject-specific MRI approach (bottom panels). Sensitivity distributions of channels intersecting and/or overlying the ROIs are shown in yellow. Those distributions that did not intersect or overlie any of the ROIs are shown in gray. Only 9 of the target ROIs have been shown for purposes of clarity in the image.

Note that a threshold value was applied to the visualization to eliminate low likelihood photon paths, typically representing scattered photons with large path lengths, which are unlikely to be received at the detector during an fNIRS recording session. We tested a range of threshold values to estimate at what threshold estimated optical density values (see below) achieved an asymptote. Threshold values of 0.01 , $0.005,0.001,0.0005,0.0001,0.00005$, and 0.00001 yielded optical density changes of $0.032,0.048,0.065,0.068,0.071,0.072$ and 0.072 units. Since an asymptote was observed from a threshold value of 0.0001 , we chose this value for all-further processing.

Overall, there is a good overlap between the target ROIs (blue) and the sensitivity distributions (yellow). Recall that this figure depicts our final geometry. Prior to arriving at this final stage, the visualization was very useful. We used this tool to determine whether all the ROIs were intersecting the sensitivity distributions (channels) and to

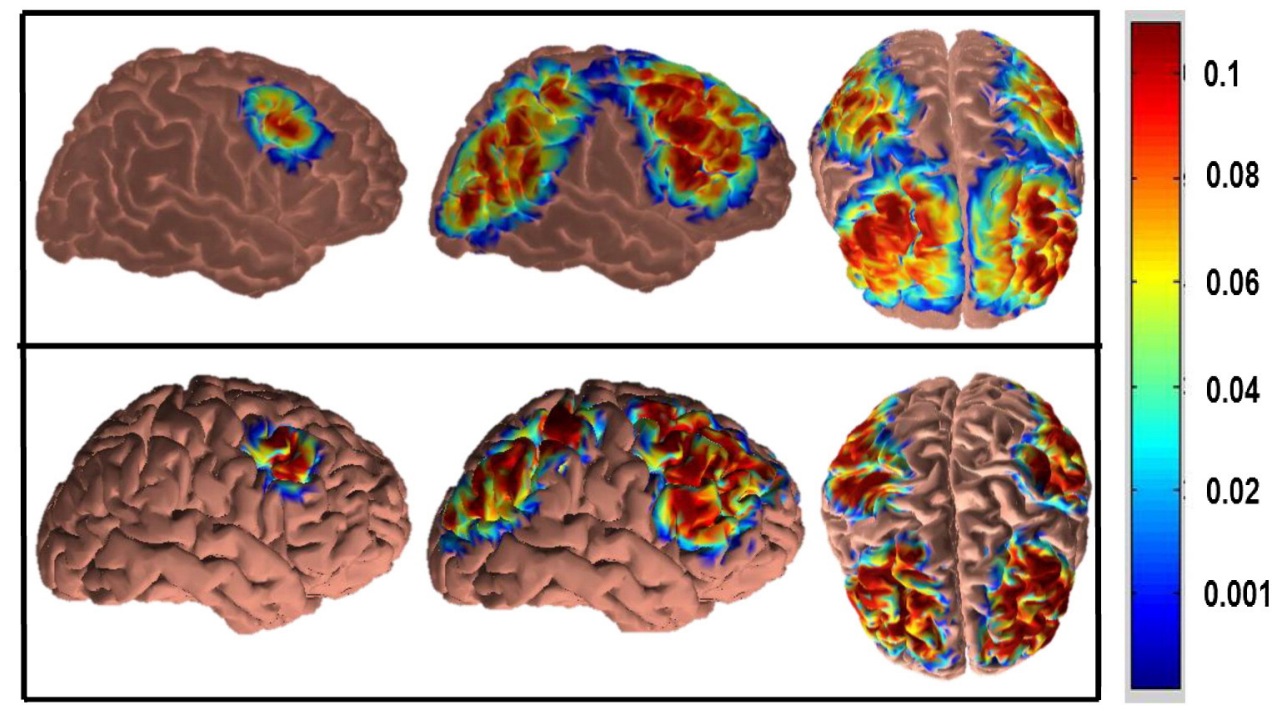

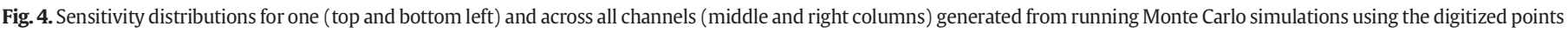

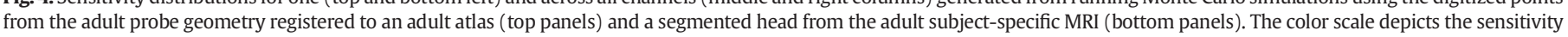
logarithmically from 0.001 to 0.1 . 


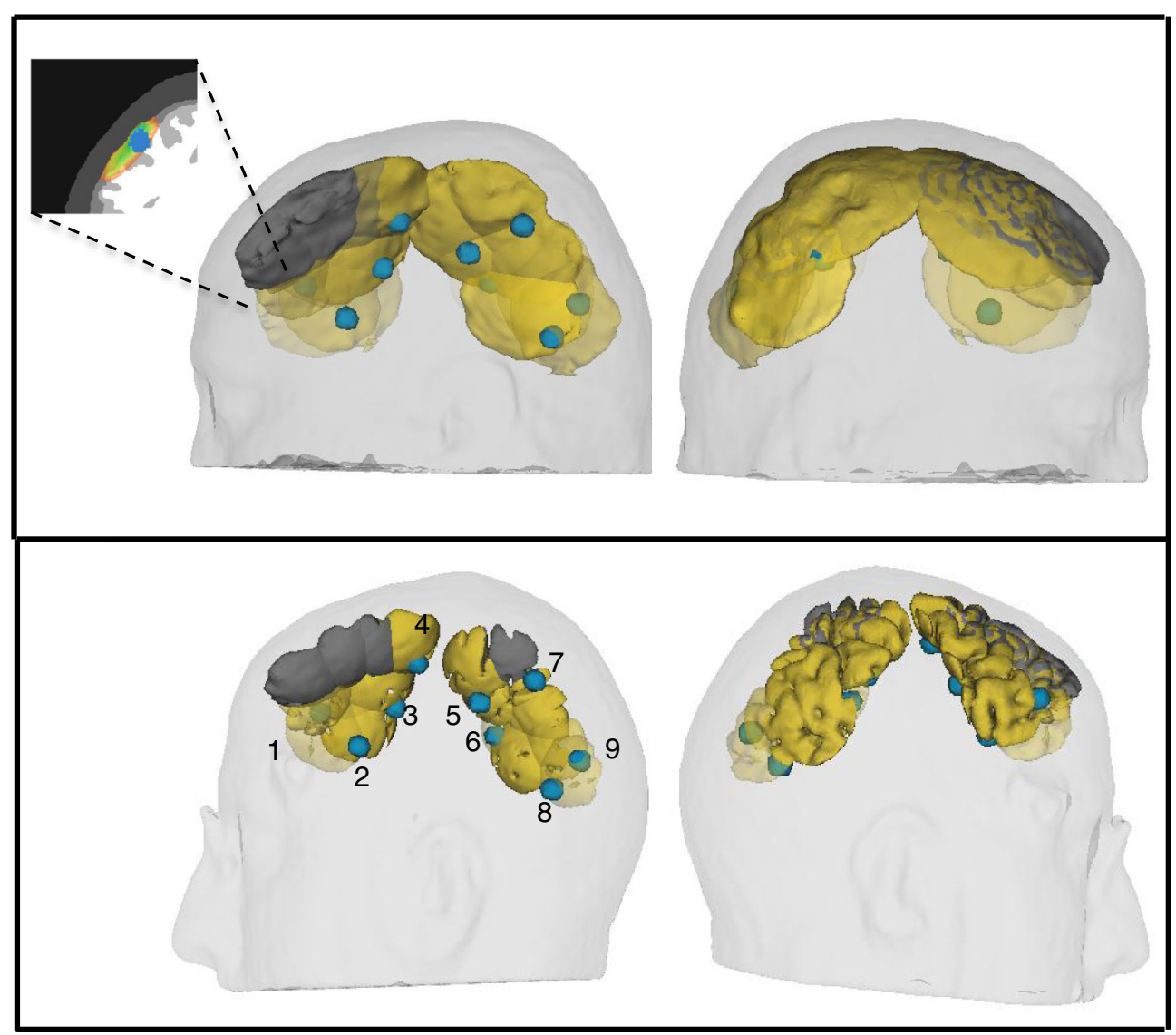

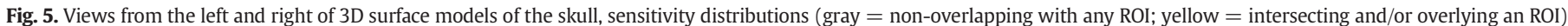

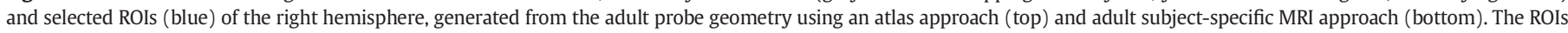

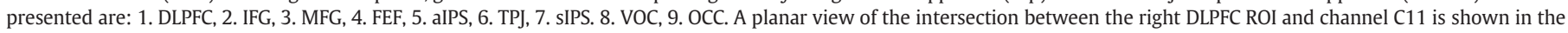
inset in the top left corner with probabilities depicted by the colors (green $=$ higher probability).

re-position channels to optimize this overlap. Once it appeared that we had a good geometry, we then moved to the next step-to quantify the amount of intersection.

Quantifying overlap between sensitivity distributions and ROIs (Step 6b). The sensitivity distributions created through the Monte Carlo photon migration simulations track the probability that photons will pass through a given brain volume as they migrate from a source to a detector. Given a random walk process, it is probable that some light will pass through the target ROIs, but what we want to quantify is the likelihood of detecting an absorption change within the intersection volume where a given channel intersects a given ROI.

We can do this by estimating the optical density change that would be measured by a source-detector pair (i.e., a channel) given an absorption change within the intersection volume. The baseline absorption of brain tissue is approximately $0.01 \mathrm{~mm}^{-1}$; thus, a robust, localized functional activation within an intersection volume might yield a $10 \%$ change in absorption $\left(0.001 \mathrm{~mm}^{-1}\right)$ as an upper limit. We can multiply this absorption change by the sensitivity of a given voxel within the intersection volume and integrate over the volume. This will yield an estimated optical density change generated by a hypothetical activation within the intersection volume. For example, if we observe a sensitivity of $0.1 \mathrm{~mm}^{-2}$ across a set of voxels within a $5 \times 5 \times 5 \mathrm{~mm}$ volume of interest, we would multiply the sensitivity $\left(0.1 \mathrm{~mm}^{-2}\right)$ by the estimated absorption change $\left(0.001 \mathrm{~mm}^{-1}\right)$ and then integrate over the volume $\left(125 \mathrm{~mm}^{3}\right)$. This would yield an optical density change of 0.0125 .

The next question is how to interpret these data. For comparison, the heartbeat typically generates an optical density change in the order of 0.01 . This signal is easily detected within raw data. With appropriate filtering and signal processing, it is likely that one could detect an optical density change 10 times smaller than this amount (i.e., 0.001 ). Thus, as a rule of thumb, intersections that yield optical density changes in the order of 0.001 should be considered to have an adequate overlap between the NIRS channel and the ROI. Note that this rule of thumb is not an absolute threshold. For instance, it is possible that one could detect optical density changes lower than 0.001 by sampling activation repeatedly over trials and modeling the resultant hemodynamic response. Moreover, the optical density changes estimated using this approach are dependent on the volume of the ROIs used, an issue we discuss in greater detail below.

Using this approach, we estimated the optical density change that would result from a localized activation within the intersection volume for each channel and ROI. Table 3 reports these data for the atlas (adult to generic atlas) and subject-specific MRI approaches (adult to adult subject-specific MRI). The serial number for each ROI corresponds with that of Table 2 . Note that very low optical density values such as those for the intersections between channels and left IFG are considered negligible and equal to zero.

Across both the adult atlas and adult subject-specific MRI approaches, 17 out of the 21 target ROIs intersected one or more channels. The ROIs that did not intersect any of the channels were the left IPS, bilateral IFG, and the left ACC (shown in red in Table 3). All these ROIs were positioned too deep into the cortex to record using fNIRS. This was true for both the adult atlas and adult subject-specific MRI approaches.

In general, there were a greater number of intersections between ROIs and channels for the adult atlas approach than for the adult subject-specific MRI approach; however, both approaches showed a 
Table 3

Optical density change $\left(10^{-5}\right.$ dimensionless units) estimated from a localized absorption change within the intersection volume for each channel and right/left hemispheric ROIs across the atlas and subject-specific MRI approaches for the adult subject. The numbers in the bracket following the ROIs correspond with that of Table 2. Those intersections shown in red did not achieve any intersections across both approaches. The ROI shown in blue intersected a channel in the generic adult-atlas but not the subject-specific MRI approach. The ROI shown in green intersected a channel in the subject-specific MRI but not the generic adult-atlas approach.

\begin{tabular}{|c|c|c|c|c|c|c|}
\hline & \multicolumn{3}{|c|}{ Right hemisphere } & \multicolumn{3}{|c|}{ Left hemisphere } \\
\hline ROI (\#) & Ch. & $\begin{array}{l}\text { Adult subject- } \\
\text { specific MRI }\end{array}$ & $\begin{array}{c}\text { Generic } \\
\text { adult atlas }\end{array}$ & Ch. & $\begin{array}{l}\text { Adult subject- } \\
\text { specific MRI }\end{array}$ & $\begin{array}{r}\text { Generic } \\
\text { adult atlas }\end{array}$ \\
\hline $\operatorname{sIPS}(1)$ & 31 & 0.01 & 0.07 & & & \\
\hline $\operatorname{sIPS}(1)$ & 33 & 0.07 & 0.08 & & & \\
\hline IPS $(2,3)$ & 33 & 0.08 & 0.07 & 26 & 0 & 0 \\
\hline $\operatorname{IPS}(2)$ & 35 & 0 & 0.38 & & & \\
\hline aIPS $(4,5)$ & 32 & 0.25 & 0 & 21 & 0.10 & 0.53 \\
\hline aIPS (5) & & & & 22 & 0 & 4.43 \\
\hline $\operatorname{aIPS}(5)$ & & & & 26 & 10.07 & 0.12 \\
\hline $\operatorname{aIPS}(5)$ & & & & 27 & 4.80 & 6.03 \\
\hline $\operatorname{VOC}(6,7)$ & 28 & 9.91 & 37.74 & 19 & 0.14 & 0.13 \\
\hline $\operatorname{VOC}(6,7)$ & 29 & 0 & 0.11 & 23 & 5.28 & 52.26 \\
\hline $\operatorname{VOC}(6,7)$ & 30 & 0 & 0.20 & 24 & 20.96 & 25.08 \\
\hline $\operatorname{VOC}(7)$ & & & & 25 & 3.95 & 64.47 \\
\hline $\operatorname{DLPFC}(8,9)$ & 10 & 3.38 & 125.01 & 1 & 25.11 & 45.50 \\
\hline $\operatorname{DLPFC}(8,9)$ & 11 & 842.76 & 917.88 & 2 & 20.72 & 431.22 \\
\hline $\operatorname{DLPFC}(8,9)$ & 12 & 0 & 9.08 & 3 & 2.90 & 1.09 \\
\hline $\operatorname{DLPFC}(8,9)$ & 18 & 0 & 1.07 & 6 & 661.80 & 97.17 \\
\hline $\operatorname{DLPFC}(8,9)$ & 15 & 19.97 & 43.56 & 7 & 155.75 & 393.63 \\
\hline $\operatorname{DLPFC}(8,9)$ & 16 & 531.01 & 224.10 & 8 & 0.25 & 0.18 \\
\hline $\operatorname{DLPFC}(8,9)$ & 17 & 0 & 0.44 & 9 & 0.61 & 3.16 \\
\hline SFG (10) & & & & 14 & 0 & 0.63 \\
\hline IFG $(11,12)$ & 1 & 0 & 0 & 1 & 0 & 0.01 \\
\hline IFG (12) & & & & 2 & 0 & 0.03 \\
\hline $\operatorname{FEF}(13,14)$ & 13 & 25.61 & 16.68 & 3 & 0 & 0.24 \\
\hline $\operatorname{FEF}(13,14)$ & 14 & 0.09 & 3.38 & 4 & 7.41 & 33.47 \\
\hline FEF (14) & & & & 5 & 0 & 5.19 \\
\hline MFG $(15,16)$ & 10 & 2.54 & 3.34 & 3 & 3.52 & 4.69 \\
\hline MFG (15) & 12 & 15.23 & 4.90 & & & \\
\hline MFG (15) & 15 & 0 & 0.09 & & & \\
\hline $\mathrm{ACC}(17)$ & 0 & 0 & 0 & & & \\
\hline $\operatorname{OCC}(18,19)$ & 28 & 150.38 & 623.84 & 19 & 0 & 2.51 \\
\hline OCC $(18,19)$ & 29 & 1.91 & 9.43 & 23 & 10.68 & 395.69 \\
\hline OCC (19) & & & & 24 & 9.00 & 55.36 \\
\hline OCC (19) & & & & 25 & 0 & 5.71 \\
\hline $\operatorname{TPJ}(20,21)$ & 29 & 0 & 3.36 & 19 & 23.53 & 80.68 \\
\hline $\operatorname{TPJ}(20,21)$ & 30 & 8.18 & 239.53 & 20 & 49.23 & 760.38 \\
\hline $\operatorname{TPJ}(20,21)$ & 33 & 0 & 1.39 & 21 & 60.41 & 22.20 \\
\hline $\operatorname{TPJ}(20,21)$ & 34 & 0 & 12.91 & 22 & 173.23 & 322.46 \\
\hline $\operatorname{TPJ}(20,21)$ & 35 & 0 & 14.28 & 25 & 0 & 24.56 \\
\hline $\operatorname{TPJ}(20,21)$ & 36 & 54.37 & 620.68 & 27 & 0.24 & 0.56 \\
\hline
\end{tabular}

considerable overlap between the sensitivity distributions and the target ROIs. There were also some unique differences between approaches. The adult subject-specific MRI approach showed a unique intersection with the right aIPS ROI (shown in green in Table 3), while the adult atlas approach showed a unique intersection with the left SFG ROI ( shown in blue in Table 3). However, these unique intersections presented with relatively small optical density changes.

Both approaches showed weak intersections with the right sIPS (see Table 3 ). Once again, this ROI was positioned relatively deep in the cortex. Note that our ROIs had radii of $6 \mathrm{~mm}$. Weak intersections do not necessarily indicate that the probe will not detect any activation from the right sIPS. Rather, the probe has limited sensitivity within the sphere defined by the ROI. In VWM experiments, by contrast, neural activation spreads across many voxels within the sIPS. When evaluating the fNIRS probe geometry, therefore, it is important to consider not just the coordinates from the fMRI literature, but also the spread of neural activation around these coordinates. This information could be explicitly accounted for by scaling the ROI radius based on fMRI results.

Note that our data also places constraints on how we interpret data from regions with a large overlap with the target ROIs. For example, the right DLPFC intersected 4 channels across both approaches. Of these four channels, $\mathrm{C} 11$ and $\mathrm{C} 16$ had the highest estimated optical density changes (C11: 842.76 and 917.88 units and C16: 531.01 and 224.01 units). Thus, functional brain activation from the right DLPFC is likely to be reflected within both channels.

Pearson's correlation was used to correlate the estimated optical density changes between the adult atlas and subject-specific MRI approaches. The correlation between the optical densities in the intersections between both approaches achieved significance $(\mathrm{r}=$ $0.77 \mathrm{p}<0.005$ ). Thus, both approaches provide comparable views of ROI intersections and optical densities.

\section{Modifying probe geometry as needed (Step 7)}

If the preliminary probe geometry did not achieve the desired intersections with the target ROIs, the channels must be re-positioned until an optimized geometry is obtained. As discussed in Step 2 (Preliminary probe geometry section), the positions of all sources and detectors were anchored to the $10-20$ coordinate system. Thus, it is necessary to specify changes in the geometry relative to the 10-20 anchor points.

With the geometry described in Step 2 and shown in Fig. 2, none of the channels intersected the left FEF and right FEF, which were posterior to the probe (the left FEF is shown by the blue sphere in the bottom left image in Fig. 6A). To create an overlap, we shifted the frontal geometry back on the head as shown in Fig. 6B. Lines were drawn from source S1 (F7) to T7 and S3 (F3) to C3. Using these lines as reference, the entire frontal geometry was moved $1 / 5$ of the distance between S1-T7 and S3-C3. The new positions of the sources and detectors and final left frontal geometry are shown in Fig. 6B. The plot at the bottom right shows the intersection of the left FEF with three channels after the geometry was modified (see Table 3 for details).

An important question is whether our final geometry creates more overlap with the target ROIs relative to other approaches. To evaluate this, we examined a previous data set we collected as part of an fNIRS validation study. The goal of the validation study was to examine VWM in adult participants using both fNIRS and fMRI. Critically, the optodes in this previous study were held within six chevron-shaped pads with 2 sources at the center and 4 detectors on the outside, creating 6 channels per pad. The pads were then positioned relative to the 10-20 landmarks over frontal, parietal, and temporal cortical regions in each hemisphere.

To evaluate the new geometry relative to the validation study, we processed the data from each participant $(N=13)$ from the prior study using our new pipeline and quantified the overlap with the target ROIs. We then compared the maximum estimated optical density change within each intersection volume for each ROI for the improved geometry reported here relative to each participant's data from the validation study. Comparisons with two participants from the validation study are shown in Table 4 . We selected these participants because they showed the maximum intersections with the ROIs (thus, these are the two 'best' participants from the previous study). For subject \#1, 7 channels showed greater optical density changes with this participant's geometry (shown in red), while 12 channels showed greater optical density changes with the new geometry. Perhaps more importantly, the new geometry which intersected with 4 ROIs was not detected with this participant's geometry. For subject \#2, only 3 channels showed greater optical density changes with this participant's geometry (shown in red), while 15 channels showed greater optical density changes with the new geometry. Moreover, the new geometry intersected with 8 ROIs not detected with this participant's geometry. Note that the variance between subjects 1 and 2 reflects variation in the orientation of the optode pads on the head, as well as differences in optode positioning due to differences in head size (we did not scale the source-detector distance in our prior study). Because the new approach reported here has optodes embedded within an EEG cap which is scaled with head circumference, the geometry is more reproducible from participant to participant. 
The final column in Table 4 shows a summary of the data across all 13 participants. For 15 ROIs, the new geometry outperformed the old geometry across the majority of participants. Three ROIs-R-sIPS, R-IPS and L-IPS-had greater optical density changes with the geometry used in the prior study. This greater coverage with the prior geometry was driven by the more central placement of the optodes in that study. Unfortunately, we were not able to create a geometry that covered all aspects of parietal cortex and still provided robust coverage of ROIs in the temporal and occipital cortices. We discuss potential improvements in our pipeline in the General discussion section that might overcome this limitation.

\section{Discussion}

Out of the 21 target ROIs identified from the fMRI literature, 17 achieved intersections with channels from an optimized probe design when registered over either an adult atlas or an adult subject-specific MRI scan. The four ROIs in the bilateral IFG, the left ACC, and the left IPS that did not achieve any intersections were either positioned too deep into the cortex to be able to record from using fNIRS or were positioned just outside the coverage of the overall geometry. There were more intersections for the adult atlas approach than for the adult subject-specific MRI approach; overall, however, both approaches were very similar.

Results from this experiment demonstrate that the methods and tools we have developed can be used to create an optimized probe geometry for an adult subject to investigate cortical activation relevant to an a priori research question. We also demonstrated tools to qualitatively and quantitatively validate the contribution of activation from each of the ROIs to each channel. Finally, we demonstrated that our optimized geometry outperformed an old geometry used in a previous

\section{Table 4}

Comparison of improved geometry using the new pipeline with an old geometry anchored to 10-20 landmarks for the 21 ROIs. Values in column 4 are maximum estimates of optical density changes from Table 3. Columns 5 and 6 show difference scores (old geometrynew geometry) comparing maximum optical densities for two participants from a previous study who had maximal intersection with the target ROIs. The final column shows the percentage of participants $(N=13)$ where the new geometry outperformed the old geometry for each ROI.

\begin{tabular}{|c|c|c|c|c|c|c|}
\hline \multirow{2}{*}{ No. } & \multirow{2}{*}{ ROIs } & \multirow{2}{*}{ Hemi } & \multirow{2}{*}{$\begin{array}{l}\text { Improved } \\
\text { geometry }\end{array}$} & \multicolumn{2}{|c|}{ Previous geometry } & \multirow{2}{*}{$\%$ Better with new } \\
\hline & & & & Subj \#1 & Subj \#2 & \\
\hline 1 & SIPS & $R$ & 0.0000008 & 0.0000364 & $\mathrm{~N} / \mathrm{A}$ & 15.4 \\
\hline 2 & IPS & $\mathrm{R}$ & 0.0000038 & 0.0000088 & $\mathrm{~N} / \mathrm{A}$ & 15.4 \\
\hline 3 & IPS & L & $\mathrm{N} / \mathrm{A}$ & 0.0000949 & 0.0000438 & 0.0 \\
\hline 4 & aIPS & $R$ & $\mathrm{~N} / \mathrm{A}$ & 0.0000013 & $\mathrm{~N} / \mathrm{A}$ & 0.0 \\
\hline 5 & aIPS & $\mathrm{L}$ & 0.0000603 & -0.0000529 & -0.0000092 & 100.0 \\
\hline 6 & VOC & $\mathrm{R}$ & 0.0003774 & $\mathrm{~N} / \mathrm{A}$ & -0.0003227 & 100.0 \\
\hline 7 & VOC & $\mathrm{L}$ & 0.0006447 & $\mathrm{~N} / \mathrm{A}$ & -0.0005778 & 100.0 \\
\hline 8 & DLPFC & $R$ & 0.0091788 & -0.0090179 & -0.0034231 & 76.9 \\
\hline 9 & DLPFC & $\mathrm{L}$ & 0.0043122 & $\mathrm{~N} / \mathrm{A}$ & -0.0043094 & 100.0 \\
\hline 10 & SFG & $\mathrm{L}$ & 0.0000063 & 0.0000102 & $\mathrm{~N} / \mathrm{A}$ & 92.3 \\
\hline 11 & IFG & $R$ & $\mathrm{~N} / \mathrm{A}$ & $\mathrm{N} / \mathrm{A}$ & $\mathrm{N} / \mathrm{A}$ & $\mathrm{N} / \mathrm{A}$ \\
\hline 12 & IFG & $\mathrm{L}$ & 0.0000003 & N/A & $\mathrm{N} / \mathrm{A}$ & 100.0 \\
\hline 13 & FEF & $\mathrm{R}$ & 0.0001668 & -0.0000386 & N/A & 100.0 \\
\hline 14 & FEF & $\mathrm{L}$ & 0.0003347 & $\mathrm{~N} / \mathrm{A}$ & $\mathrm{N} / \mathrm{A}$ & 100.0 \\
\hline 15 & MFG & $R$ & 0.0000490 & 0.0001149 & $\mathrm{~N} / \mathrm{A}$ & 76.9 \\
\hline 16 & MFG & $\mathrm{L}$ & 0.0000469 & 0.0001174 & $\mathrm{~N} / \mathrm{A}$ & 76.9 \\
\hline 17 & ACC & $\mathrm{L}$ & $\mathrm{N} / \mathrm{A}$ & $\mathrm{N} / \mathrm{A}$ & $\mathrm{N} / \mathrm{A}$ & $\mathrm{N} / \mathrm{A}$ \\
\hline 18 & OCC & $R$ & 0.0062384 & -0.0062379 & 0.0033180 & 92.3 \\
\hline 19 & OCC & $\mathrm{L}$ & 0.0039569 & $\mathrm{~N} / \mathrm{A}$ & 0.0010684 & 92.3 \\
\hline 20 & TPJ & $\mathrm{R}$ & 0.0062068 & -0.0006057 & -0.0058558 & 100.0 \\
\hline 21 & TPJ & $\mathrm{L}$ & 0.0076038 & -0.0053389 & -0.0038372 & 100.0 \\
\hline
\end{tabular}

study. In the sections that follow, we evaluate the generality of these tools by examining their effectiveness in early development and with a second probe geometry designed to capture motor and language processing.
A
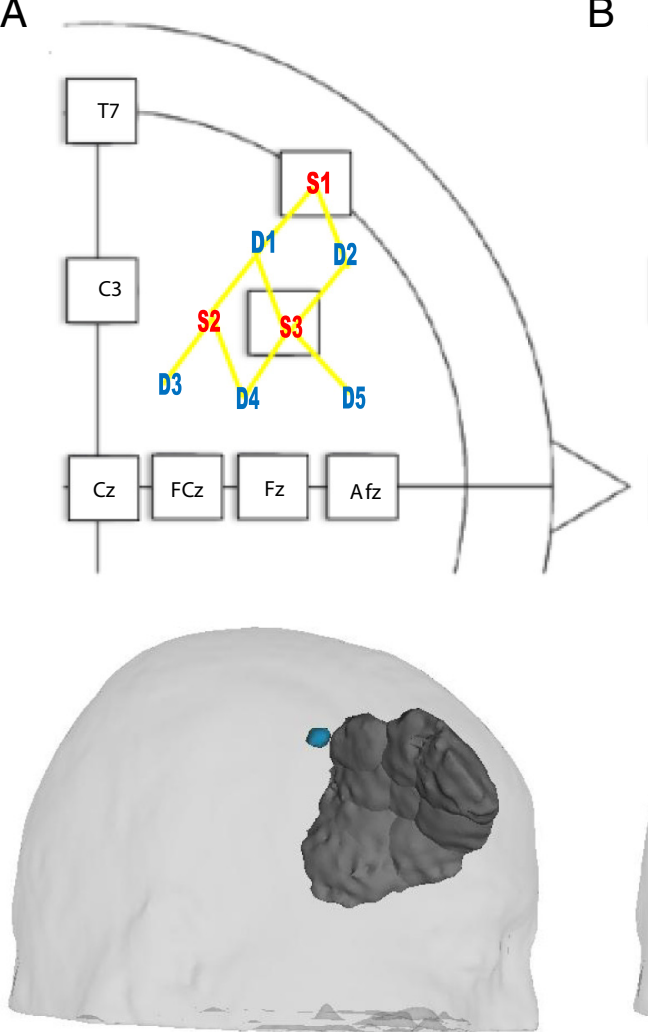

B
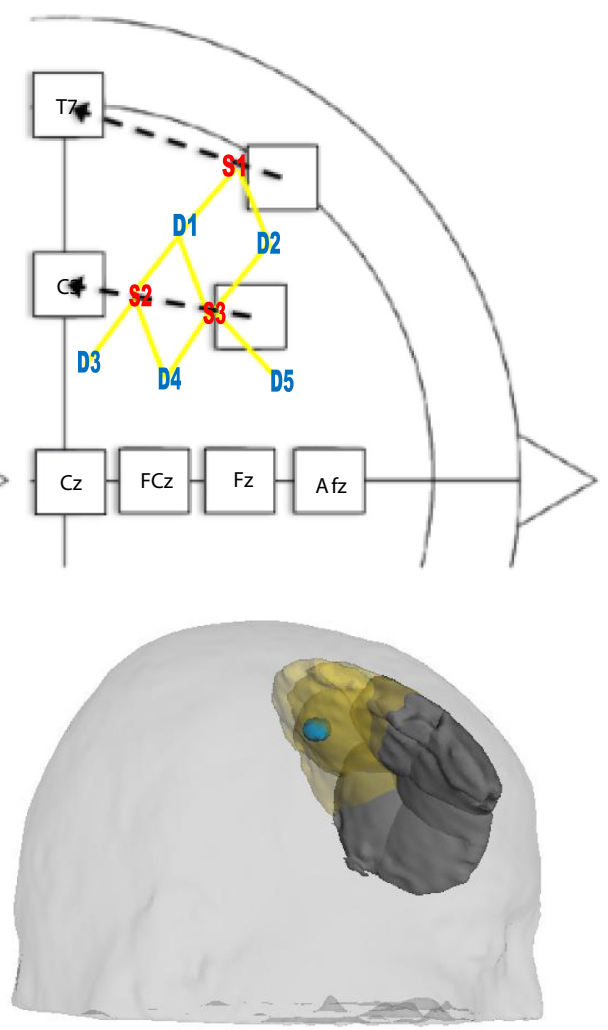

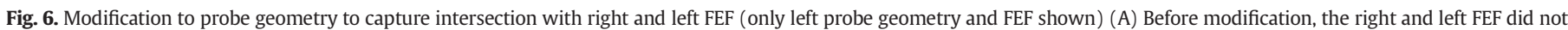

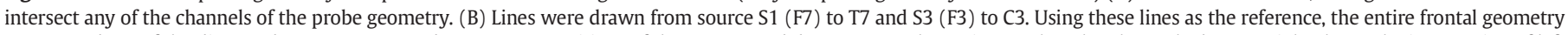

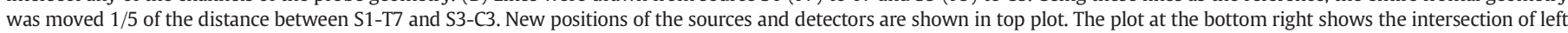
FEF with three channels after the geometry was modified. 


\section{Experiment 2}

The aim of this experiment was to scale the VWM adult probe geometry from Experiment 1 to a child's head. Digitized points from the child subject were used to transform the generic adult atlas as well as a segmented head obtained from a child subject-specific MRI scan. Note that we used the adult atlas because it was readily available as a part of the AtlasViewerGUI software. We wanted to ascertain whether using an adult atlas for a child's digitization would lead to substantial differences in the estimated overlap between ROIs and sensitivity distributions relative to using a child subject-specific MRI scan.

\section{Methods and results}

Steps 1-7 of Experiment 1 (see Fig. 1) were carried out as described previously, with one modification: we adjusted the source-detector distance to an appropriate size based on head circumference. Typically, fNIRS researchers use a source-detector distance of $30 \mathrm{~mm}$ for adult subjects, and a source-detector distance of $20 \mathrm{~mm}$ for infants. Although a fixed source-detector distance across studies has created some uniformity, fixing this distance across variations in head circumference means that the probe geometry is moving relative to the underlying anatomy across subjects. To reduce this source of variance, we created a mapping between source-detector distance and head circumference.

To establish this mapping, we consulted WHO child growth standards (Van den Broeck et al., 2009), as well as a study of centiles for adult head circumference (Bushby et al., 1992). From these sources, we constructed Table 5. This table maps head circumference in centimeters to age for both males and females. At the upper end of this range is a large male adult head with a circumference of $60 \mathrm{~cm}$; at the lower end of this range is a newborn infant head with a circumference of $38 \mathrm{~cm}$. Given that most fNIRS studies with adults use a $30 \mathrm{~mm}$ sourcedetector distance, we assigned this distance to the $60 \mathrm{~cm}$ head circumference. Next, given that most fNIRS studies with infants use a $20 \mathrm{~mm}$ source-detector distance (Taga et al., 2007), we assigned this distance to the $40 \mathrm{~cm}$ head circumference. Note that we did not use the newborn head circumference $(38 \mathrm{~cm}$ ) as a lower anchor point since many studies in infancy are with slightly older infants (e.g., 4-5 month olds). Finally, we linearly scaled the source-detector distances between the adult and infant anchor points. Table 5 shows the scaling of the source-detector separation as a function of head/cap size.

In the current experiment, we collected data from a male child (age 3 years) with a head size of $50 \mathrm{~cm}$. Thus, we scaled the VWM probe geometry down by keeping the same anchor points relative to the 10-20 system, but reducing the source-detector distance to $25 \mathrm{~mm}$ (see Table 5).

T1-weighted volumetric scans were collected using the same scan parameters as used for obtaining the adult MRI scans.

\section{Table 5}

List of head sizes and corresponding source-detector distances for males and females of all ages.

\begin{tabular}{llll}
\hline $\begin{array}{l}\text { Head size/cap } \\
\text { size }(\mathrm{cm})\end{array}$ & $\begin{array}{l}\text { Source-detector } \\
\text { distance }(\mathrm{mm})\end{array}$ & $\begin{array}{l}\text { Ages/size } \\
\text { (Years, months })\end{array}$ & \\
\cline { 2 - 4 } & & Male & Female \\
\hline 60 & 30 & Adult & - \\
58 & 29 & Medium adult & Adult \\
56 & 28 & Small adult & Medium adult \\
54 & 27 & 9 years & Small adult \\
52 & 26 & 6 years & 8 years \\
50 & 25 & 3 years 7 months & 5 years \\
48 & 24 & 1 years 10 month & 2 years 6 months \\
46 & 23 & 1 year & 1 year 5 months \\
44 & 22 & 7 months & 9 months \\
42 & 21 & 4 months & 6 months \\
40 & 20 & 2 months & 4 months \\
38 & 19 & Newborn & Newborn \\
\hline
\end{tabular}

Visualization in Slicer

Fig. 7 shows left and right views of 3D surface models of the skull, sensitivity distributions (in gray and yellow using a threshold of 0.0001 ; see Quantifying overlap between sensitivity distributions and ROIs (Step 6b)), and several ROIs (blue) of the right hemisphere for the adult atlas approach (top panels) and child subject-specific MRI approach (bottom left and right). Sensitivity distributions of channels intersecting and/or overlying the ROIs are shown in yellow. Those channels that did not intersect or overlie any of ROIs are shown in gray. The ROIs shown are arranged in the same sequence as in Fig. 5. Once again, the final geometry achieves good overlap with the target ROIs.

\section{Quantifying overlap between fNIRS recording and ROIs}

Table 6 quantifies the intersection between the sensitivity distributions and ROIs in terms of estimated optical density changes generated by an absorption change within the intersection volume for the adult atlas and child subject-specific MRI approaches. The serial numbers for each ROI correspond with that of Table 2. Again, very low optical density changes such as those for the intersections between channels and left IFG are considered negligible and equal to zero.

Across both approaches, 19 out of 21 ROIs achieved intersection with one or more channels. The left IFG and left ACC were too deep and therefore did not intersect with channels in either approach (shown in red in Table 6). The right IFG did not intersect with any channel in the adult atlas approach (shown in green in Table 6). However, this unique difference had low estimated optical density values.

On the other hand, the right OCC ROI did not intersect any of the channels in the child subject-specific MRI approach but had a large intersection with channels from the geometry used to transform the adult atlas (shown in blue in Table 6). In our view, this difference is problematic. Based on the adult atlas, we would erroneously conclude that the geometry is optimized to record from right occipital cortex; however, the child subject-specific MRI data suggest otherwise. Note that we were not able to obtain better coverage of the right OCC ROI even with more iterations through the processing pipeline due to constraints in the geometry: this area is too far away from other ROIs to cover with the limited number of channels we had available. Even though there is this substantive difference between approaches, significantly robust correlations were observed for the optical density values estimated across intersections for both approaches $(r=0.92$, $\mathrm{p}<0.005$ ).

\section{Discussion}

The aim of the second experiment was to extend and apply the tools for designing probe geometries to investigate VWM in early development. The methods and tools used were identical to those used in the previous experiment, but we scaled the adult geometry down to a child's head size. This required that we re-create the geometry anchored to 10-20 landmarks with a scaled-down source-detector distance (see Table 5). Out of the 21 target ROIs, 19 intersected channels of both approaches. The ROIs in the left ACC and the left IFG were too deep to intersect with channels. In the child subject-specific MRI approach, which presents a true representation of photon migration patterns in a child's brain, geometric constraints on the parieto-occipital probe geometry did not allow for any intersections with the OCC ROI. On the other hand, the adult atlas showed ample intersection. This is one example where the use of a generic adult atlas to map photon migration patterns in a child's brain can be misleading. Although in general, the adult atlas approach yielded comparable intersection results, we recommend that structural scans of children should be used whenever possible because this yields more accurate photon migration results. In the future, it is likely that child subject-specific atlases will be more readily available. Future work will need to investigate whether an 


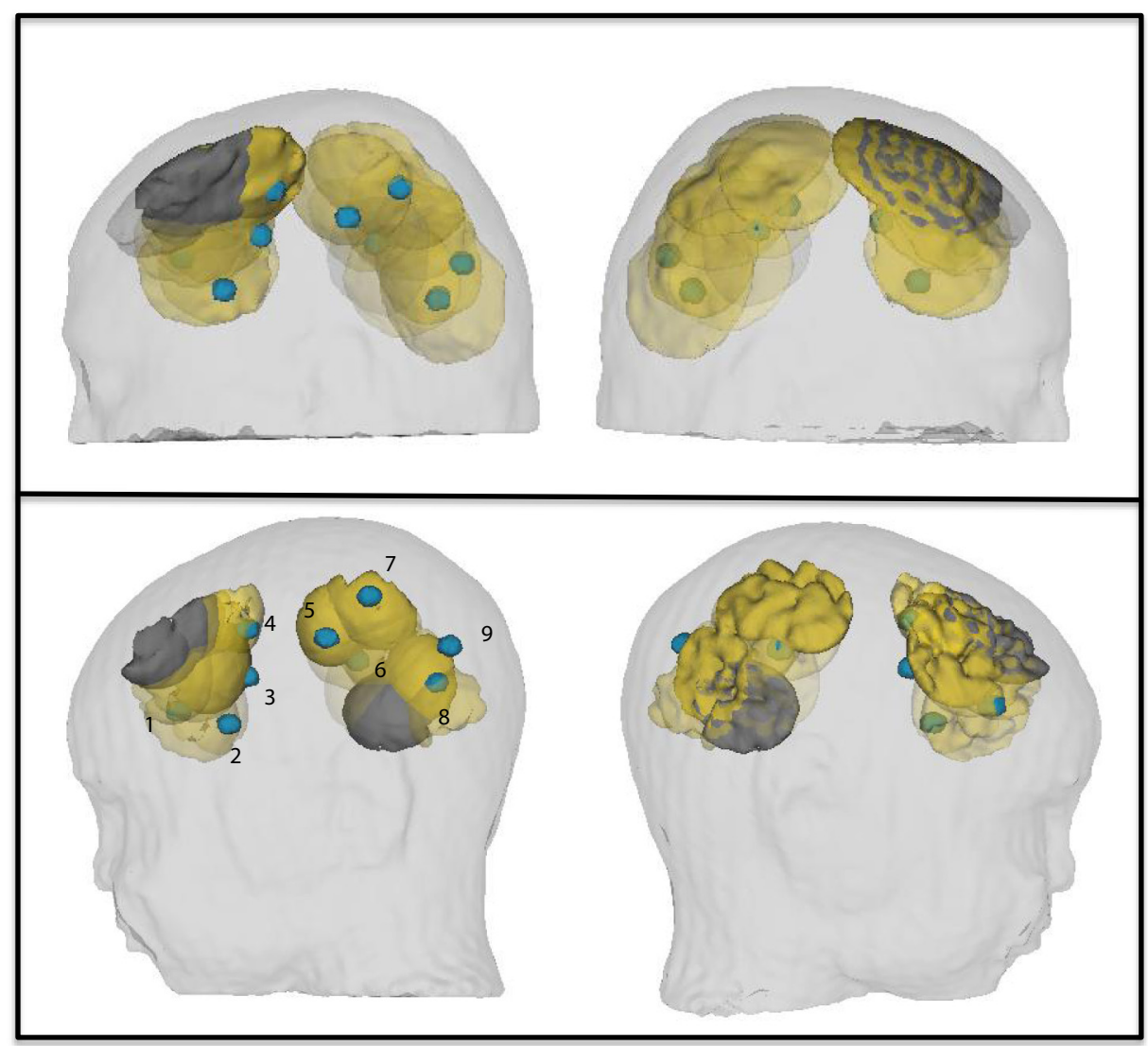

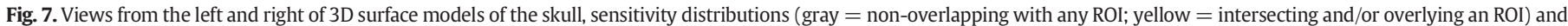

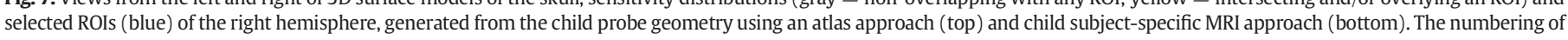
the ROIs follows the numbering from Fig. 5.

age-specific atlas yields results more consistently comparable to the child subject-specific MRI approach.

Finally, if we compare results from the adult and child probe geometries (Experiments 1 and 2), it is evident that the distribution and positioning of channels and ROIs are quite similar despite the dramatic changes in head circumference. A good example is that of the right DLPFC that achieved large intersections with the same channels across both geometries and using the adult atlas and subject-specific MRI approaches. Moreover, as expected and quite consistently reported in the literature, the thinner scalp and skull of the child's head produced much larger optical density changes as compared to the adult probe geometry. The child geometry also intersected a larger number of ROIs. For instance, the right IFG intersected a couple of channels of the child probe geometry, whereas this ROI was too deep in the adult brain. This provides a good validation of our method of scaling the probe geometry over development.

\section{Experiment 3}

The aim of this experiment was to use the developed set of tools to create an optimized probe design for a different project examining language and motor processing to determine whether the processing pipeline generalizes beyond the domain of VWM.

\section{Methods and results}

Steps 1-7 of Experiment 1 (see Fig. 1) were carried out with the exception that only the adult atlas approach was adopted. We chose a female adult (age 26 years) with a head size of $54 \mathrm{~cm}$ and an appropriate source-detector distance of $27 \mathrm{~mm}$ (see Table 5). We designed a probe geometry such that channels would intersect 47 ROIs taken from 15 articles focused on aspects of language and motor processing relevant to questions of interest in our laboratory (see Table 7). The left panel of Fig. 8 shows the digitized points from the adult subject from the left hemisphere registered onto the adult atlas. Red and blue circles represent sources and detectors and their connections are represented in yellow. The right panel of Fig. 8 shows the left hemispheric view of the sensitivity distributions for all the channels.

\section{Visualization in Slicer and quantifying overlap between fNIRS recording and ROIs}

Fig. 9 shows views from the left and right, of 3D surface models of the skull, sensitivity distributions (in gray and yellow using a threshold of 0.0001; see Quantifying overlap between sensitivity distributions and ROIs (Step 6b)), and several ROIs (blue) of the right hemisphere. Sensitivity distributions of channels intersecting and/or overlying the ROIs are shown in yellow. Those channels that did not intersect or overlie any of the ROIs are shown in gray. Table 8 quantifies the intersection between the sensitivity distributions and ROIs in terms of estimated optical density changes generated by an absorption change within the intersection volume.

Of the 47 ROIs, 35 ROIs were covered by the probe geometry. The left superior temporal gyrus, bilateral Insula, right putamen, left thalamus, right cerebellum, left cingulate and bilateral medial premotor cortex were located too deep to be covered by any fNIRS geometry and therefore excluded from further consideration. The ROIs in the right Anterior Ventrolateral Prefrontal Cortex and bilateral Visual Association-Superior Occipital ROIs were outside of the coverage of the probe geometry.

Unlike the probe geometry in Experiments 1 and 2 which were designed around four quadrants, the current design included one expansive cluster that moved from lateral frontal and temporal regions 
Table 6

Optical density change ( $10^{-5}$ dimensionless units) estimated from a localized absorption change within the intersection volume for each channel and left/right hemispheric ROIs across the adult atlas and child subject-specific MRI approaches. The serial numbers of the ROIs corresponds with that of Table 2. Intersections shown in red did not achieve any intersections across both approaches. The ROI shown in blue intersected a channe in the generic adult-atlas approach but not the child subject-specific MRI approach. The ROI shown in green intersected a channel in the child subject-specific MRI approach but not the generic adult-atlas approach.

\begin{tabular}{|c|c|c|c|c|c|c|}
\hline & & Right hemi & & & Left hemisph & \\
\hline ROI (\#) & Ch. & $\begin{array}{l}\text { Child subject- } \\
\text { specific MRI }\end{array}$ & $\begin{array}{c}\text { Generic } \\
\text { adult atlas }\end{array}$ & Ch. & $\begin{array}{l}\text { Child subject- } \\
\text { specific MRI }\end{array}$ & $\begin{array}{l}\text { Generic } \\
\text { adult atlas }\end{array}$ \\
\hline $\operatorname{sIPS}(1)$ & 31 & 2.67 & 21.19 & & & \\
\hline $\operatorname{sIPS}(1)$ & 33 & 0 & 0.35 & & & \\
\hline IPS $(2,3)$ & 31 & 12.77 & 22.39 & 26 & 2.43 & 3.28 \\
\hline $\operatorname{IPS}(2)$ & 33 & 0.01 & 2.22 & & & \\
\hline aIPS $(4,5)$ & 32 & 2.79 & 3.52 & 22 & 0.13 & 4.73 \\
\hline $\operatorname{aIPS}(4,5)$ & 34 & 0 & 0.34 & 26 & 0.40 & 9.54 \\
\hline aIPS (5) & & & & 27 & 29.32 & 21.88 \\
\hline $\operatorname{VOC}(6,7)$ & 28 & 44.05 & 498.40 & 19 & 136.99 & 0.77 \\
\hline $\operatorname{VOC}(6,7)$ & 29 & 26.28 & 9.42 & 20 & 0.57 & 0.12 \\
\hline $\operatorname{VOC}(6,7)$ & 30 & 0 & 1.58 & 21 & 0.18 & 0 \\
\hline $\operatorname{VOC}(7)$ & & & & 23 & 0.17 & 115.15 \\
\hline $\operatorname{VOC}(7)$ & & & & 24 & 320.51 & 133.01 \\
\hline $\operatorname{VOC}(7)$ & & & & 25 & 0 & 30.13 \\
\hline $\operatorname{DLPFC}(8,9)$ & 10 & 82.86 & 91.89 & 1 & 0.63 & 4.02 \\
\hline $\operatorname{DLPFC}(8,9)$ & 11 & 3411.43 & 1117.13 & 2 & 366.13 & 337.71 \\
\hline $\operatorname{DLPFC}(8,9)$ & 12 & 0.44 & 17.56 & 6 & 0 & 6.48 \\
\hline $\operatorname{DLPFC}(8,9)$ & 14 & 0 & 0.10 & 7 & 34.01 & 132.78 \\
\hline $\operatorname{DLPFC}(8,9)$ & 18 & 0 & 0.51 & 9 & 0 & 0.68 \\
\hline $\operatorname{DLPFC}(8)$ & 15 & 64.83 & 54.77 & & & \\
\hline $\operatorname{DLPFC}(8)$ & 16 & 292.25 & 284.40 & & & \\
\hline SFG (10) & & & & 14 & 0 & 1.79 \\
\hline SFG (10) & & & & 17 & 0.06 & 1.43 \\
\hline IFG $(11,12)$ & 10 & 0.11 & 0 & 1 & 0 & 0.01 \\
\hline $\operatorname{IFG}(11,12)$ & 11 & 0.52 & 0 & 2 & 0 & 0.03 \\
\hline $\operatorname{FEF}(13,14)$ & 13 & 61.54 & 16.54 & 4 & 41.34 & 33.28 \\
\hline $\operatorname{FEF}(13,14)$ & 14 & 2.04 & 1.37 & 5 & 0.49 & 0.77 \\
\hline MFG $(15,16)$ & 10 & 0.17 & 1.65 & 1 & 0.14 & 0.28 \\
\hline MFG $(15,16)$ & 12 & 17.85 & 4.84 & 3 & 2.05 & 3.87 \\
\hline MFG $(15,16)$ & 15 & 4.14 & 1.06 & 4 & 0 & 0.26 \\
\hline MFG (16) & & & & 6 & 0.23 & 0.21 \\
\hline $\mathrm{ACC}(17)$ & & 0 & 0 & & & \\
\hline $\operatorname{OCC}(18,19)$ & 28 & 0 & 271.35 & 19 & 1.68 & 1.03 \\
\hline $\operatorname{OCC}(18,19)$ & 29 & 0 & 15.11 & 23 & 0 & 68.85 \\
\hline $\mathrm{OCC}(19)$ & & & & 24 & 3.51 & 35.73 \\
\hline $\operatorname{TPJ}(20,21)$ & 29 & 0 & 5.73 & 19 & 3.43 & 131.75 \\
\hline $\operatorname{TPJ}(20,21)$ & 30 & 0 & 23.04 & 20 & 27.36 & 693.83 \\
\hline $\operatorname{TPJ}(20,21)$ & 31 & 0 & 1.45 & 21 & 14.21 & 97.83 \\
\hline $\operatorname{TPJ}(20,21)$ & 32 & 1.93 & 1.96 & 22 & 1934.06 & 558.59 \\
\hline $\operatorname{TPJ}(20,21)$ & 33 & 760.44 & 124.19 & 24 & 0 & 2.19 \\
\hline TPJ $(20,21)$ & 34 & 1708.77 & 154.52 & 25 & 0 & 6.34 \\
\hline TPJ $(20,21)$ & 35 & 420.68 & 142.88 & 26 & 1.15 & 6.12 \\
\hline TPJ $(20,21)$ & 36 & 1577.82 & 563.22 & 27 & 107.25 & 17.95 \\
\hline
\end{tabular}

up to the motor strip. With this type of geometry, a single channel was expected to intersect multiple ROIs. For example, channel 23 intersects with the left Dorsolateral Prefrontal Cortex, left Middle Ventrolateral Prefrontal Cortex, and the left Triangularis/Inferior Frontal Gyrus. The advantage of quantifying intersections is evident in this case, because we can determine from Table 8 that channel 23 largely reflects activation of the left Middle Ventrolateral Prefrontal Cortex - this region has the highest optical densities followed by the left Triangularis/Inferior Frontal Gyrus. Thus, the table provides a guide for interpreting patterns of activation across channels and the likely source of such patterns in cortex.

\section{Discussion}

The purpose of this final experiment was to demonstrate that our processing pipeline could be generalized to a new probe geometry targeting a different set of ROIs. As is evident in Fig. 9, the probe geometry was optimized such that all channels were utilized and we obtained the maximal coverage given constraints on the total number of channels available. Results demonstrate that our processing pipeline can be
Table 7

List of ROIs used in the design of the probe for language and motor processing (Experiment 3). The serial numbering for each ROI is used for identification in later tables.

\begin{tabular}{|c|c|c|c|c|c|}
\hline \multirow[t]{2}{*}{ No. } & \multirow[t]{2}{*}{ Regions of interest } & \multirow[t]{2}{*}{ Hemipshere } & \multicolumn{3}{|c|}{ MNI coordinates } \\
\hline & & & $\mathrm{x}$ & $\mathrm{y}$ & z \\
\hline 1 & Inferior Frontal Gyrus & Right & 52 & 12 & -8 \\
\hline 2 & Inferior Frontal Gyrus & Left & -41 & 21 & 7 \\
\hline 3 & Middle Frontal Gyrus & Left & -46 & 10 & 43 \\
\hline 4 & Middle Frontal Gyrus & Right & 39 & 7 & 52 \\
\hline 5 & Superior Frontal Gyrus & Right & 14 & 16 & 52 \\
\hline 6 & Supplementary Motor Area & Right & 4 & 9 & 76 \\
\hline 7 & Supplementary Motor Area & Left & -6 & 2 & 54 \\
\hline 8 & Precentral & Right & 58 & 2 & 29 \\
\hline 9 & Precentral & Left & -37 & 7 & 35 \\
\hline 10 & Inferior Parietal Lobule & Left & -29 & -47 & 52 \\
\hline 11 & Inferior Parietal Lobule & Right & 45 & -46 & 49 \\
\hline 12 & Superior Temporal Gyrus & Left & -37 & -33 & 12 \\
\hline 13 & Superior Temporal Gyrus & Right & 57 & -31 & 9 \\
\hline 14 & Insula & Right & 44 & -16 & 3 \\
\hline 15 & Insula & Left & -39 & -17 & 4 \\
\hline 16 & Thalamus & Left & -18 & -19 & 5 \\
\hline 17 & Putamen & Right & 29 & -4 & 5 \\
\hline 18 & Cerebellum & Right & 13 & -58 & -14 \\
\hline 19 & Cingulate & Left & -8 & -19 & 45 \\
\hline 20 & Operacularis/Ventral Premotor & Right & 54 & -4 & 28 \\
\hline 21 & Operacularis/Ventral Premotor & Left & -61 & -4 & 27 \\
\hline 22 & Primary Motor Cortex & Right & 40 & -15 & 49 \\
\hline 23 & Primary Motor Cortex & Left & -40 & -15 & 49 \\
\hline 24 & Lateral Precentral Gyrus & Right & 45 & 15 & 45 \\
\hline 25 & Lateral Precentral Gyrus & Left & -45 & 15 & 45 \\
\hline 26 & Triangularis/Inferior Frontal Gyrus & Right & 54 & 40 & 8 \\
\hline 27 & Triangularis/Inferior Frontal Gyrus & Left & -50 & 44 & 10 \\
\hline 28 & Dorsolateral Prefrontal Cortex & Right & 45 & 42 & 21 \\
\hline 29 & Dorsolateral Prefrontal Cortex & Left & -42 & 41 & 20 \\
\hline 30 & Middle Ventrolateral Prefrontal Cortex & Right & 54 & 29 & 10 \\
\hline 31 & Middle Ventrolateral Prefrontal Cortex & Left & -54 & 29 & 10 \\
\hline 32 & Primary Somatosensory/Postcentral & Right & 56 & -20 & 36 \\
\hline 33 & Primary Somatosensory/Postcentral & Left & -48 & -30 & 62 \\
\hline 34 & Supramarginal Gyrus & Right & 48 & -32 & 41 \\
\hline 35 & Supramarginal Gyrus & Left & -58 & -30 & 36 \\
\hline 36 & Superior Parietal Lobule & Right & 12 & -53 & 72 \\
\hline 37 & Superior Parietal Lobule & Left & -23 & -61 & 67 \\
\hline 38 & Visual Association-Superior Occipital & Right & 20 & -86 & 27 \\
\hline 39 & Visual Association-Superior Occipital & Left & -18 & -94 & 20 \\
\hline 40 & Dorsal Premotor/Dorsal Precentral Gyrus & Right & 24 & -7 & 59 \\
\hline 41 & Dorsal Premotor/Dorsal Precentral Gyrus & Left & -32 & -8 & 56 \\
\hline 42 & Medial Premotor & Right & 6 & -2 & 52 \\
\hline 43 & MEDIAL PREMOTOR & Left & -6 & -2 & 52 \\
\hline 44 & Anterior Ventrolateral Prefrontal Cortex & Right & 50 & 32 & -12 \\
\hline 45 & Anterior Ventrolateral Prefrontal Cortex & Left & -50 & 32 & -12 \\
\hline 46 & Intraparietal Sulcus & Right & 28 & -54 & 57 \\
\hline 47 & Intraparietal Sulcus & Left & -29 & -54 & 58 \\
\hline
\end{tabular}

effectively used to design, visualize, and quantify the details across a wide range of probe geometries.

\section{General discussion}

In the past few decades, fNIRS studies have become increasingly frequent, investigating cortical function in typical and atypical infants, children, and adults (Boas et al., 2014). Moreover, recent papers have refined signal processing methods for fNIRS to address motion filtering and artifact reduction (Brigadoi et al., 2014). Nevertheless, relatively little focus has been placed upon the design and optimization of probe geometry to record from cortical ROIs relevant to specific research questions. Rather, most studies use probes that are broadly distributed across an entire cortical area of interest. This may not be ideal in some research contexts, particularly given constraints on the number of fNIRS channels available. To address these concerns, we described a new processing pipeline to accurately capture activation from target ROIs and optimize probe geometry to better utilize the available fNIRS channels. 


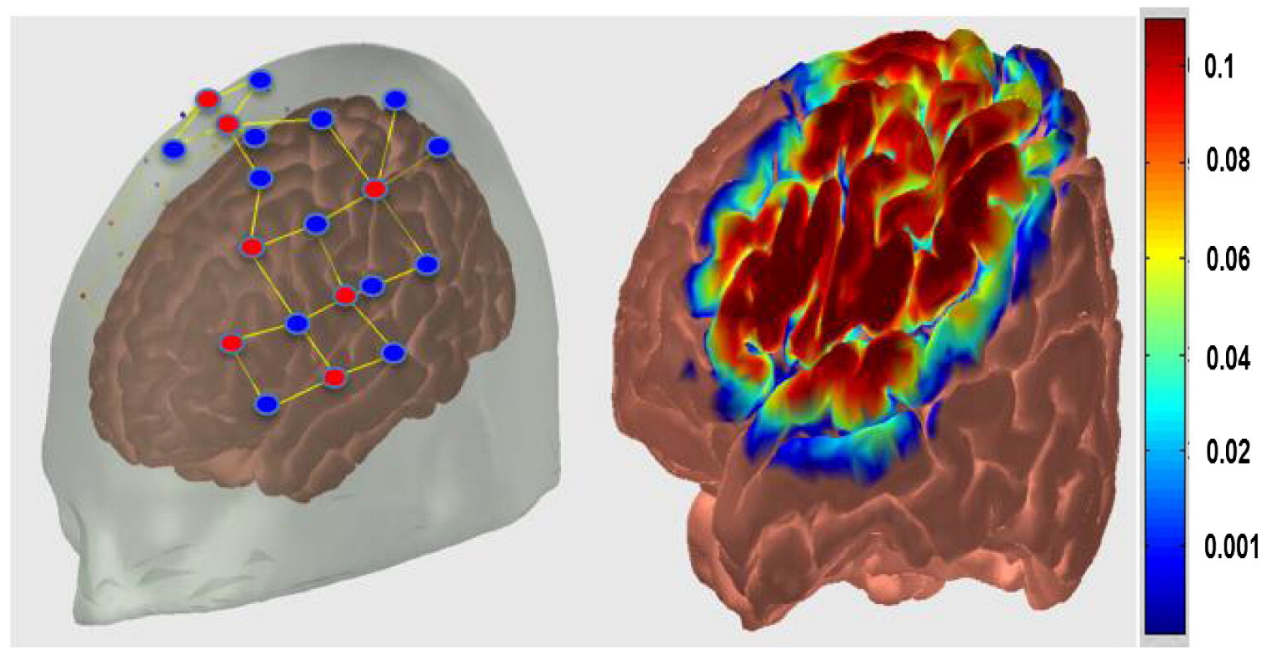

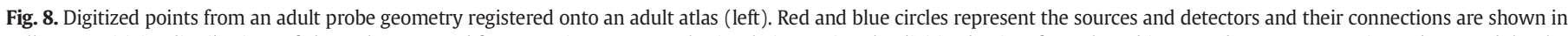

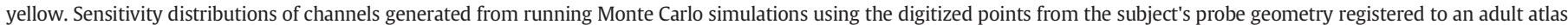
(right). The color scale depicts the sensitivity logarithmically from 0.001 to 0.1 .

In the first two experiments, we developed a methodology and an accompanying set of tools to create an optimized probe geometry for studying VWM in adults and children. Across experiments, we used a subject-specific MRI and an adult atlas approach to assess the generality of our methods across subjects (adult and child).

In experiment 1 , the probe geometry was designed such that it could capture activation from many of the ROIs from an adult subject whose scalp landmarks were transformed to fit a generic adult atlas. The adult atlas is readily and freely available and is, therefore, the best option in the absence of resources for subjects' structural scans. To validate our processing pipeline and to investigate how accurately the results from the adult atlas are represented by the subject's true anatomy, we also used a segmented head created from his own MRI scan.

We demonstrated that out of the 21 target ROIs, all except the left ACC, bilateral IFG, and left IPS intersected channels across both approaches. The subject-specific MRI approach did not achieve as many intersections as the adult-atlas approach; however, this is likely a true reflection of photon absorption within cortical matter. In the absence of individualized anatomical information, our results demonstrate that an adult atlas can be used effectively. Critically, quantified data on intersections and estimated optical density changes can be used with both approaches to guide the interpretation of channelbased analyses.
In the second experiment, we modified the probe geometry such that the distance between the sources and detectors was scaled down to fit a child subject. Once again, we used the adult atlas transformed to the child's scalp landmarks and also a segmented head obtained from the child's MRI scan to examine if the results from both approaches were comparable. Out of the 21 target ROIs, only the left ACC and the left IFG did not intersect any channel from either approach. Thus, we obtained excellent coverage of the target ROIs. Similar to the observations made in Experiment 1, channels from the child subject-specific MRI approach intersected fewer ROIs than the adult atlas approach. However, in one particular case - the right OCC ROI - there was a discrepancy across approaches. The subject-specific MRI approach is an accurate representation of photon migration distributions in the child's brain and within this context, the right OCC ROI was positioned outside of the constraints of the probe geometry. This is incorrectly represented in an adult atlas where the digitized points from a child's head are forced to fit an adult's head. Given this, we recommend that segmented head volumes obtained from subject-specific MRI scans should be used whenever possible. An alternative, cost-effective approach would be to use age-specific atlases. Future work will be needed to compare whether results obtained from an age-specific atlas would be better than that obtained from a generic adult atlas.

Considered together, results of Experiments 1 and 2 demonstrate the effectiveness of our processing pipeline when generalizing across

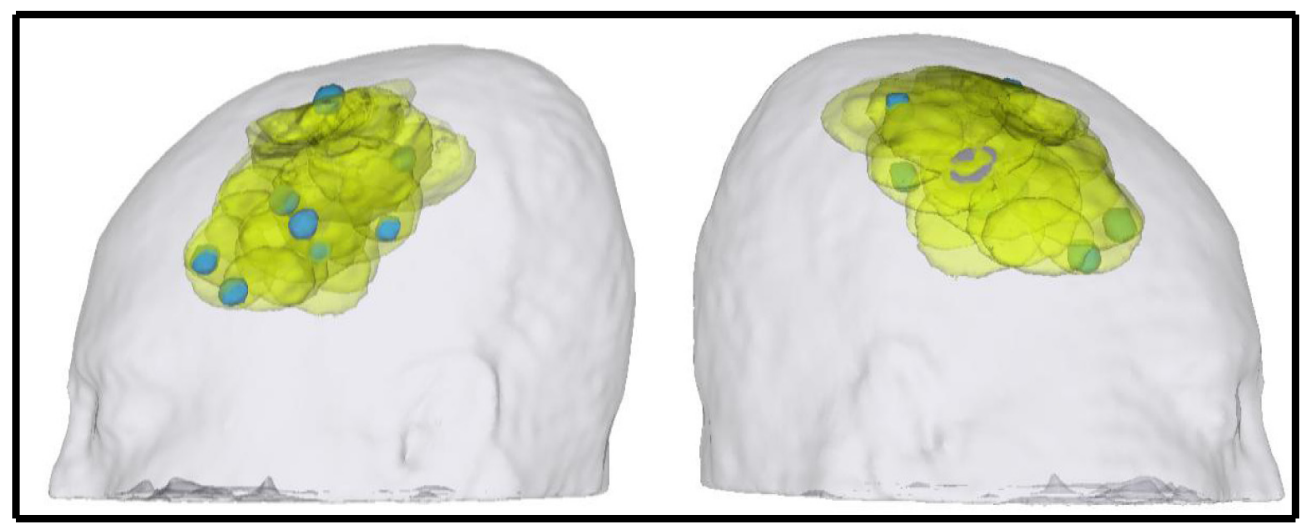

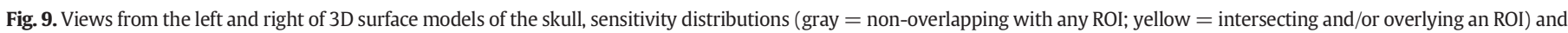
selected ROIs (blue) of the right hemisphere, generated from the adult probe geometry using an atlas approach. 
Table 8

Only intersections between sensitivity distributions and left and right hemispheric ROIs with maximum optical densities $\left(\times 10^{-5}\right.$ dimensionless units $)$ shown. The serial numbers of the ROIs correspond with that of Table 7.

\begin{tabular}{|c|c|c|c|c|}
\hline No. & ROI & Hemi & Ch. & $\Delta \mathrm{OD}$ \\
\hline 1 & Inferior Frontal Gyrus & $\mathrm{R}$ & 36 & 0.40 \\
\hline 2 & Inferior Frontal Gyrus & $\mathrm{L}$ & 13 & 9.16 \\
\hline 3 & Middle Frontal Gyrus & $\mathrm{L}$ & 19 & 731.75 \\
\hline 4 & Middle Frontal Gyrus & $\mathrm{R}$ & 44 & 247.72 \\
\hline 5 & Superior Frontal Gyrus & $\mathrm{R}$ & 41 & 21.43 \\
\hline 6 & Supplementary Motor Area & $\mathrm{R}$ & 37 & 539.74 \\
\hline 7 & Supplementary Motor Area & $\mathrm{L}$ & 37 & 1.04 \\
\hline 8 & Precentral & $\mathrm{R}$ & 32 & 1923.87 \\
\hline 9 & Precentral & $\mathrm{L}$ & 20 & 6.94 \\
\hline 10 & Inferior Parietal Lobule & $\mathrm{L}$ & 5 & 2.08 \\
\hline 11 & Inferior Parietal Lobule & $\mathrm{R}$ & 24 & 187.10 \\
\hline 13 & Superior Temporal Gyrus & $\mathrm{R}$ & 35 & 1.22 \\
\hline 20 & Operacularis/Ventral Premotor & $\mathrm{R}$ & 32 & 1473.53 \\
\hline 21 & Operacularis/Ventral Premotor & $\mathrm{L}$ & 9 & 209.80 \\
\hline 22 & Primary Motor Cortex & $\mathrm{R}$ & 27 & 133.89 \\
\hline 23 & Primary Motor Cortex & $\mathrm{L}$ & 3 & 52.64 \\
\hline 24 & Lateral Precentral Gyrus & $\mathrm{R}$ & 42 & 1020.86 \\
\hline 25 & Lateral Precentral Gyrus & $\mathrm{L}$ & 21 & 1078.10 \\
\hline 26 & Triangularis/Inferior Frontal Gyrus & $\mathrm{R}$ & 46 & 51.12 \\
\hline 27 & Triangularis/Inferior Frontal Gyrus & $\mathrm{L}$ & 23 & 863.72 \\
\hline 28 & Dorsolateral Prefrontal Cortex & $\mathrm{R}$ & 45 & 397.15 \\
\hline 29 & Dorsolateral Prefrontal Cortex & $\mathrm{L}$ & 23 & 228.95 \\
\hline 30 & Middle Ventrolateral Prefrontal Cortex & $\mathrm{R}$ & 46 & 274.62 \\
\hline 31 & Middle Ventrolateral Prefrontal Cortex & $\mathrm{L}$ & 23 & 1264.36 \\
\hline 32 & Primary Somatosensory/Postcentral & $\mathrm{R}$ & 28 & 1404.52 \\
\hline 33 & Primary Somatosensory/Postcentral & $\mathrm{L}$ & 2 & 220.46 \\
\hline 34 & Supramarginal Gyrus & $\mathrm{R}$ & 29 & 243.44 \\
\hline 35 & Supramarginal Gyrus & $\mathrm{L}$ & 2 & 50.87 \\
\hline 36 & Superior Parietal Lobule & $\mathrm{R}$ & 24 & 1.69 \\
\hline 37 & Superior Parietal Lobule & $\mathrm{L}$ & 5 & 3.35 \\
\hline 40 & Dorsal Premotor/Dorsal Precentral Gyrus & $\mathrm{R}$ & 26 & 104.70 \\
\hline 41 & Dorsal Premotor/Dorsal Precentral Gyrus & $\mathrm{L}$ & 14 & 161.88 \\
\hline 45 & Anterior Ventrolateral Prefrontal Cortex & $\mathrm{L}$ & 13 & 41.74 \\
\hline 46 & Intraparietal Sulcus & $\mathrm{R}$ & 24 & 10.02 \\
\hline 47 & Intraparietal Sulcus & $\mathrm{L}$ & 5 & 3.51 \\
\hline
\end{tabular}

the dramatic differences in head size one naturally confronts in developmental studies. Using the newly developed tools, we were able to verify a priori that we could record from the target ROIs in both 3 -year-olds and adults and quantify the resultant intersections at each age. Some differences across ages emerged such as differences in the distribution of overlap across channels and differences in the number of ROIs that intersected the sensitivity volumes. Importantly, our method quantifies these differences in a way that can help guide the interpretation of results in developmental studies.

In the final experiment, we used the same tools to generate a different probe geometry for another study where the ROIs were more closely spaced. Once again, we achieved an overlap with many of the ROIs. Some were too deep to intersect with the channels. Other ROIs were spread across over regions too far away to be covered by the scope of our geometry given the number of NIRS channels we had access to. Given the close proximity of most ROIs and the continuous nature of our geometry, there were multiple ROIs that intersected a single channel. This highlighted another advantage of our approach: quantification of overlap and optical density changes can help determine which ROIs are likely to impact resultant hemodynamic differences and identify cases where differences are likely to be ambiguous. Specifically, a difference in activation at a channel elicited by a specific experimental condition and/or contrast can be accurately pinpointed to the ROI with the largest optical density change.

It is important to take note of possible sources of error in this methodology. A previous work by Singh et al. identified that the variability of scalp to brain projections across subjects was between 4 and $7 \mathrm{~mm}$ (Singh et al., 2005). Further, Cooper et al. estimated that error in Euclidean distances in the localization of brain activity due to issues such as differences in gyral/sulcal patterns increased from
9.1 $\mathrm{mm}$ when using the subjects' MRI scans to $18.0 \mathrm{~mm}$ when using the atlas based approach (Cooper et al., 2012). These observations are consistent with our findings that the subject-specific MRI approach provides a more accurate representation of the underlying anatomy and photon migration patterns than the adult atlas approach.

There are at least two ways in which future efforts might improve upon the processing pipeline we presented here. It may be possible to use mathematical optimization techniques to maximize an overlap between sensitivity volumes and ROIs. For instance, one could simulate variations in a local geometry and select the geometry that maximizes intersection and simulated optical density changes. The challenge would be to reconstruct the resultant geometry on a real, physical cap. This was the limiting factor in our design work-we always had to start with a reproducible geometry and work from there. It might be possible, however, to remove this constraint using modeling software to map the resultant geometry onto a model EEG cap and print the result using $3 \mathrm{D}$ printing techniques.

An alternative approach would be to digitize a dense array of possible points in a large probe geometry, go through the steps of the processing pipeline, and then select the geometry that maximizes intersection and optical densities. This would substantially reduce the number of alterations and iterations required for optimizing the positions of the sources and detectors; however, depending on the density of the array, it may create substantial computational demands. Moreover, the initial dense array would have to be specified in a way that the selected geometry could be readily reproduced on a physical cap.

Another future effort would be to use sophisticated meta-analytical approaches in the fMRI literature like Activation Likelihood Estimation to accurately identify and model ROIs as the center of a Gaussian probability distribution which can be summed to create a statistical image of the likelihood of activation of each voxel across and within experiments (Laird et al., 2009; Turkeltaub et al., 2012). This creates a more systematic method for accumulating ROIs across relevant contrasts, experiments, and studies.

A final future direction is to use the registration methods developed here to move the analysis of fNIRS data from channel-space to a voxelbased image space commonly used in fMRI analytical designs. For instance, one could fit a GLM model to fNIRS data, creating a $\beta$-map for each NIRS channel. The sensitivity volume for each channel from photon migration could then be weighted by these $\beta$ values to construct a voxel-based functional image for each experimental condition.

\section{Acknowledgments}

We would like to thank Shelby Putt and Bryan Brown for their help in designing the probe geometry for Experiment 3.

JPS acknowledges support from NSF HSD0527698.

DAB acknowledges support from NIH P41 EB015896.

Note that DAB is an inventor on a technology licensed to TechEn, a company whose medical pursuits focus on noninvasive, optical brain monitoring. DAB's interests were reviewed and are managed by Massachusetts General Hospital and Partners HealthCare in accordance with their conflict of interest policies.

\section{Conflict of interest}

The authors have no conflict of interest.

\section{References}

Alloway, T.P., 2007. Working memory, reading, and mathematical skills in children with developmental coordination disorder. J. Exp. Child Psychol. 96, 20-36.

Boas, D.A., Elwell, C.E., Ferrari, M., Taga, G., 2014. Twenty years of functional near-infrared spectroscopy: introduction for the special issue. Neurolmage 85 (Pt 1), 1-5.

Bortfeld, H., Wruck, E., Boas, D.A., 2007. Assessing infants' cortical response to speech using near-infrared spectroscopy. NeuroImage 34, 407-415. 
Brigadoi, S., Ceccherini, L., Cutini, S., Scarpa, F., Scatturin, P., Selb, J., Gagnon, L., Boas, D.A Cooper, R.J., 2014. Motion artifacts in functional near-infrared spectroscopy: a comparison of motion correction techniques applied to real cognitive data. Neurolmage 85 (Pt 1), 181-191.

Bushby, K.M., Cole, T., Matthews, J.N., Goodship, J.A., 1992. Centiles for adult head circumference. Arch. Dis. Child. 67, 1286-1287.

Buss, A.T., Fox, N., Boas, D.A., Spencer, J.P., 2014. Probing the early development of visual working memory capacity with functional near-infrared spectroscopy. Neurolmage 85 (Pt 1), 314-325.

Cooper, R.J., Caffini, M., Dubb, J., Fang, Q., Custo, A., Tsuzuki, D., Fischl, B., Wells 3rd, W., Dan, I., Boas, D.A., 2012. Validating atlas-guided DOT: a comparison of diffuse optical tomography informed by atlas and subject-specific anatomies. NeuroImage 62, 1999-2006.

Cui, X., Bray, S., Bryant, D.M., Glover, G.H., Reiss, A.L., 2011. A quantitative comparison of NIRS and fMRI across multiple cognitive tasks. Neurolmage 54, 2808-2821.

Custo, A., Boas, D.A., Tsuzuki, D., Dan, I., Mesquita, R., Fischl, B., Grimson, W.E., Wells III, W., 2010. Anatomical atlas-guided diffuse optical tomography of brain activation. Neurolmage 49, 561-567.

Fang, Q., Boas, D.A., 2009. Monte Carlo simulation of photon migration in 3D turbid media accelerated by graphics processing units. Opt. Express 17, 20178-20190.

Fava, E., Hull, R., Baumbauer, K., Bortfeld, H., 2013. Hemodynamic responses to speech and music in preverbal infants. Child Neuropsychol.

Fedorov, A., Beichel, R., Kalpathy-Cramer, J., Finet, J., Fillion-Robin, J.-C., Pujol, S., C. B., Jennings, D., Fennessy, F., Sonka, M., Buatti, J., Aylward, S.R., Miller, J.V., Pieper, S., Kikinis, R., 2012. 3D Slicer as an image computing platform for the quantitative imaging network. Magn. Reson. Imaging 1323-1341.

Gagnon, L., Yucel, M.A., Dehaes, M., Cooper, R.J., Perdue, K.L., Selb, J., Huppert, T.J., Hoge, R.D., Boas, D.A., 2012. Quantification of the cortical contribution to the NIRS signal over the motor cortex using concurrent NIRS-fMRI measurements. NeuroImage 59, 3933-3940.

Gervain, J., Mehler, J., Werker, J.F., Nelson, C.A., Csibra, G., Lloyd-Fox, S., Shukla, M., Aslin, R.N., 2011. Near-infrared spectroscopy: a report from the McDonnell infant methodology consortium. Dev. Cogn. Neurosci. 1, 22-46.

Huppert, T.J., Hoge, R.D., Diamond, S.G., Franceschini, M.A., Boas, D.A., 2006. A temporal comparison of BOLD, ASL, and NIRS hemodynamic responses to motor stimuli in adult humans. Neurolmage 29, 368-382.

Huppert, T.J., Diamond, S.G., Franceschini, M.A., Boas, D.A., 2009. HomER: a review of time-series analysis methods for near-infrared spectroscopy of the brain. Appl. Opt. 48, D280-D298.

Jausovec, N., Jausovec, K., 2012. Working memory training: improving intelligencechanging brain activity. Brain Cogn. 79, 96-106.

Kovelman, I., Shalinsky, M.H., White, K.S., Schmitt, S.N., Berens, M.S., Paymer, N., Petitto, L.A., 2009. Dual language use in sign-speech bimodal bilinguals: fNIRS brainimaging evidence. Brain Lang. 109, 112-123.

Kuboyama, N., Nabetani, T., Shibuya, K., Machida, K., Ogaki, T., 2004. The effect of maximal finger tapping on cerebral activation. J. Physiol. Anthropol. Appl. Hum. Sci. 23, 105-110.

Laird, A.R., Eickhoff, S.B., Kurth, F., Fox, P.M., Uecker, A.M., Turner, J.A., Robinson, J.L., Lancaster, J.L., Fox, P.T., 2009. ALE meta-analysis workflows via the brainmap database: progress towards a probabilistic functional brain atlas. Front. Neuroinform. $3,23$.

Laird, A.R., Robinson, J.L., McMillan, K.M., Tordesillas-Gutierrez, D., Moran, S.T. Gonzales, S.M., Ray, K.L., Franklin, C., Glahn, D.C., Fox, P.T., Lancaster, J.L., 2010 Comparison of the disparity between Talairach and MNI coordinates in functional neuroimaging data: validation of the Lancaster transform. NeuroImage 51 677-683.

Lin, P.Y., Roche-Labarbe, N., Dehaes, M., Fenoglio, A., Grant, P.E., Franceschini, M.A., 2013. Regional and hemispheric asymmetries of cerebral hemodynamic and oxygen metabolism in newborns. Cereb. Cortex 23, 339-348.

Sato, Y., Uzuka, T., Aoki, H., Natsumeda, M., Oishi, M., Fukuda, M., Fujii, Y., 2012. Nearinfrared spectroscopic study and the Wada test for presurgical evaluation of expressive and receptive language functions in glioma patients: with a case report of dissociated language functions. Neurosci. Lett. 510, 104-109.

Sato, $H$., Yahata, N., Funane, T. Takizawa, $R$, Katura, T. Atsumori, $H$., Nishimura, $Y$, Kinoshita, A., Kiguchi, M., Koizumi, H., Fukuda, M., Kasai, K., 2013. A NIRS-fMRI investigation of prefrontal cortex activity during a working memory task. NeuroImage 83 158-173.

Selb, J., Ogden, T.M., Dubb, J., Fang, Q., Boas, D.A., 2014. Comparison of a layered slab and an atlas head model for Monte Carlo fitting of time-domain near-infrared spectroscopy data of the adult head. J. Biomed. Opt. 19, 16010.

Singh, A.K., Okamoto, M., Dan, H., Jurcak, V., Dan, I., 2005. Spatial registration of multichannel multi-subject fNIRS data to MNI space without MRI. NeuroImage 27, 842-851.

Steele, S.D., Minshew, N.J., Luna, B., Sweeney, J.A., 2007. Spatial working memory deficits in autism. J. Autism Dev. Disord. 37, 605-612.

Taga, G., Homae, F., Watanabe, H., 2007. Effects of source-detector distance of near infrared spectroscopy on the measurement of the cortical hemodynamic response in infants. NeuroImage 38, 452-460.

Turkeltaub, P.E., Eickhoff, S.B., Laird, A.R., Fox, M., Wiener, M., Fox, P., 2012. Minimizing within-experiment and within-group effects in activation likelihood estimation meta-analyses. Hum. Brain Mapp. 33, 1-13.

Van den Broeck, J., Willie, D., Younger, N., 2009. The World Health Organization child growth standards: expected implications for clinical and epidemiological research. Eur. J. Pediatr. 168, 247-251.

Whalen, C., Maclin, E.L., Fabiani, M., Gratton, G., 2008. Validation of a method for coregistering scalp recording locations with 3D structural MR images. Hum. Brain Mapp. 29, 1288-1301.

Wijeakumar, S., Shahani, U., McCulloch, D.L., Simpson, W.A., 2012a. Neural and vascular responses to fused binocular stimuli: a VEP and fNIRS study. Invest. Ophthalmol. Vis. Sci. 53, 5881-5889.

Wijeakumar, S., Shahani, U., Simpson, W.A., McCulloch, D.L., 2012b. Localization of hemodynamic responses to simple visual stimulation: an fNIRS study. Invest. Ophthalmol. Vis. Sci. 53, 2266-2273.

Yoo, S., Lee, K.M., 2013. Articulation-based sound perception in verbal repetition: a functional NIRS study. Front. Hum. Neurosci. 7, 540. 